TRANSACTIONS OF THE

AMERICAN MATHEMATICAL SOCIETY

Volume 361, Number 10, October 2009, Pages 5061-5088

S 0002-9947(09)04921-6

Article electronically published on May 18, 2009

\title{
VARIETIES WITH DEFINABLE FACTOR CONGRUENCES
}

\author{
PEDRO SÁNCHEZ TERRAF AND DIEGO J. VAGGIONE
}

\begin{abstract}
We study direct product representations of algebras in varieties. We collect several conditions expressing that these representations are definable in a first-order-logic sense, among them the concept of Definable Factor Congruences (DFC). The main results are that DFC is a Mal'cev property and that it is equivalent to all other conditions formulated; in particular we prove that $\mathcal{V}$ has DFC if and only if $\mathcal{V}$ has $\overrightarrow{0} \& \overrightarrow{1}$ and Boolean Factor Congruences. We also obtain an explicit first-order definition $\Phi$ of the kernel of the canonical projections via the terms associated to the Mal'cev condition for DFC, in such a manner that it is preserved by taking direct products and direct factors. The main tool is the use of central elements, which are a generalization of both central idempotent elements in rings with identity and neutral complemented elements in a bounded lattice.
\end{abstract}

\section{INTRODUCTION}

An algebra is a nonempty set together with an arbitrary but fixed collection of finitary operations. A variety is an equationally-definable class of algebras over the same language. A congruence of an algebra $A$ is the kernel $\{(a, b) \in A: f(a)=$ $f(b)\}$ of a homomorphism $f$ with domain $A$; it is a factor congruence of $A$ if $f$ is a projection onto a direct factor of $A$. Thus, a direct product representation is determined by the pair of complementary factor congruences given by the canonical projections.

In this universal-algebraic setting, one key concept for the deeper study of direct product representations is that of central element. This tool can be developed fruitfully in varieties with $\overrightarrow{0} \& \overrightarrow{1}$, which we now define.

A variety with $\overrightarrow{0} \& \overrightarrow{1}$ is a variety $\mathcal{V}$ in which there exist unary terms $0_{1}(w), \ldots$, $0_{l}(w), 1_{1}(w), \ldots, 1_{l}(w)$ such that

$$
\mathcal{V} \models \overrightarrow{0}(w)=\overrightarrow{1}(w) \rightarrow x=y,
$$

where $w, x$ and $y$ are distinct variables, $\overrightarrow{0}=\left(0_{1}, \ldots, 0_{l}\right)$ and $\overrightarrow{1}=\left(1_{1}, \ldots, 1_{l}\right)$.

The terms $\overrightarrow{0}$ and $\overrightarrow{1}$ are analogous, in a rather general manner, to identity (top) and null (bottom) elements in rings (lattices), and its existence in a variety is equivalent to the fact that no nontrivial algebra in the variety has a trivial subalgebra. Throughout this paper we will assume that $\mathcal{V}$ is a variety with $\overrightarrow{0} \& \overrightarrow{1}$ such that the terms $\overrightarrow{0}$ and $\overrightarrow{1}$ are closed. Of course, this can be achieved when the language has a constant symbol, and we will make this assumption in order to simplify and clarify our treatment. The proofs remain valid in the general case.

Received by the editors December 15, 2006.

2000 Mathematics Subject Classification. Primary 08B05; Secondary 03C40.

This work was supported by CONICET and SECYT-UNC.

(C)2009 American Mathematical Society Reverts to public domain 28 years from publication 
If $\vec{a} \in A^{l}$ and $\vec{b} \in B^{l}$, we will write $[\vec{a}, \vec{b}]$ in place of $\left(\left(a_{1}, b_{1}\right), \ldots,\left(a_{l}, b_{l}\right)\right) \in$ $(A \times B)^{l}$. If $A \in \mathcal{V}$, we say that $\vec{e} \in A^{l}$ is a central element of $A$ if there exists an isomorphism $A \rightarrow A_{1} \times A_{2}$ such that

$$
\vec{e} \mapsto[\overrightarrow{0}, \overrightarrow{1}] .
$$

Two central elements $\vec{e}, \vec{f}$ will be called complementary if there exists an isomorphism $A \rightarrow A_{1} \times A_{2}$ such that $\vec{e} \mapsto[\overrightarrow{0}, \overrightarrow{1}]$ and $\vec{f} \mapsto[\overrightarrow{1}, \overrightarrow{0}]$.

Central elements are a generalization of both central idempotent elements in rings with identity and neutral complemented elements in a bounded lattice. It is well known that in the classical cases, central elements are powerful tools since they translate the concepts of factor congruence and of direct product representation into first-order logic. In the general framework of an arbitrary variety $\mathcal{V}$ with $\overrightarrow{0} \& \overrightarrow{1}$, we may write this definability property in the following fashion:

there exists a first-order formula $\Phi(x, y, \vec{z}, \vec{w})$ in the language of $\mathcal{V}$ such that for all $A, B \in \mathcal{V}$, and $a, c \in A, b, d \in B$,

$$
A \times B \models \Phi((a, b),(c, d),[\overrightarrow{0}, \overrightarrow{1}],[\overrightarrow{1}, \overrightarrow{0}]) \quad \text { if and only if } \quad a=c .
$$

It was shown in 12 that in varieties with $\overrightarrow{0} \& \overrightarrow{1}$ that have the Fraser-Horn-Hu property, central elements have this property, and the formula $\Phi$ may be chosen of the form $\exists \wedge p=q$. Moreover, when the variety is congruence modular, $\Phi$ can be chosen to be a conjunction of equations. One last family of varieties with $\overrightarrow{0} \& \overrightarrow{1}$ (which generalize the above-mentioned examples) is that of varieties in which factor congruences are compact [13].

Which is the most general context in which central elements concentrate the information concerning the direct product representations? One answer is given by the following condition: each pair of complementary central elements determines uniquely a pair of complementary factor congruences associated to them. This property may be written as:

for $A \in \mathcal{V}$, the map

$$
\begin{aligned}
& \left(\theta, \theta^{*}\right) \mapsto \text { unique }(\vec{e}, \vec{f}) \in A^{l} \times A^{l} \text { satisfying } \overrightarrow{0} \theta \vec{e} \theta^{*} \overrightarrow{1} \text { and } \\
& \overrightarrow{1} \theta \vec{f} \theta^{*} \overrightarrow{0}
\end{aligned}
$$

is a bijection between the set of pairs of complementary factor congruences of $A$ and the set of pairs of complementary central elements of $A$.

Another important concept involved in this paper is that of an algebra with Boolean Factor Congruences (BFC), that is, an algebra in which the set of factor congruences is a distributive sublattice of its congruence lattice. Though it is not apparent, this concept is intimately connected to the direct product construction. In the classical work of Chang, Jónsson and Tarski [3] it is proved that BFC is equivalent to the strict refinement property (a strengthening of the property that states that every two direct product representations have a common refinement). It is also noteworthy that in several works on sheaf representations 2, 4, 5, 9, $\mathrm{BFC}$ has played an important role. For example, in Bigelow and Burris [1] it is shown that in a variety with $\mathrm{BFC}$ the Boolean product representations with directly indecomposable factors are unique and coincide with the Pierce sheaf [10. We refer the reader to the work of Willard [14] as a key reference on BFC. 
In the present work, we will prove that all the conditions mentioned in this introduction are indeed equivalent for the class of varieties with $\overrightarrow{0} \& \overrightarrow{1}$.

Theorem 1.1. Let $\mathcal{V}$ be a variety with $\overrightarrow{0} \& \overrightarrow{1}$. The following are equivalent:

$(1) \mathcal{V}$ has the Weak Determining Property: for $A \in \mathcal{V}$, the map

$$
\left(\theta, \theta^{*}\right) \mapsto \text { unique }(\vec{e}, \vec{f}) \in A^{l} \times A^{l} \text { satisfying } \overrightarrow{0} \theta \vec{e} \theta^{*} \overrightarrow{1} \text { and } \overrightarrow{1} \theta \vec{f} \theta^{*} \overrightarrow{0}
$$

is a bijection between the set of pairs of complementary factor congruences of $A$ and the set of pairs of complementary central elements of $A$.

$(2) \mathcal{V}$ has the Determining Property: For $A \in \mathcal{V}$, the map

$$
\left(\theta, \theta^{*}\right) \mapsto \text { unique } \vec{e} \in A^{l} \text { satisfying } \overrightarrow{0} \theta \vec{e} \theta^{*} \overrightarrow{1}
$$

is a bijection between the set of pairs of complementary factor congruences of $A$ and the set of central elements of $A$.

$(3) \mathcal{V}$ has Definable Factor Congruences (DFC): There exists a first-order formula $\Phi(x, y, \vec{z})$ in the language of $\mathcal{V}$ such that for all $A, B \in \mathcal{V}$, and $a, c \in A$, $b, d \in B$,

$$
A \times B=\Phi((a, b),(c, d),[\overrightarrow{0}, \overrightarrow{1}]) \quad \text { if and only if } \quad a=c .
$$

(4) There exists a first-order formula $\Phi(x, y, \vec{z}, \vec{w})$ in the language of $\mathcal{V}$ such that for all $A, B \in \mathcal{V}$, and $a, c \in A, b, d \in B$,

$$
A \times B \models \Phi((a, b),(c, d),[\overrightarrow{0}, \overrightarrow{1}],[\overrightarrow{1}, \overrightarrow{0}]) \quad \text { if and only if } \quad a=c .
$$

(5) $\mathcal{V}$ has $B F C$.

Moreover, when the above equivalent conditions hold, the formula $\Phi$ in (3) can be chosen to be preserved by direct products and direct factors and for every $A \in \mathcal{V}$, the map

$$
\vec{e} \mapsto \Phi^{A}(\cdot, \cdot, \vec{e})
$$

is a bijection between the set of central elements and the Boolean algebra of factor congruences of $A$.

We now briefly sketch the contents of each section. In Section 2 we give a Mal'cev condition for a Left Determining Property to be defined there; this condition is entirely analogous to a Mal'cev condition for BFC. The terms obtained in this section are the building blocks for our definability constructions. Section 3 provides an explicit formula $\Phi$ satisfying (3) of Theorem [1.1. This formula is constructed in such a way that it is preserved by direct products and direct factors; this last assertion is proved in the Appendix. In Section 4, we characterize in first-order logic the (pairs of complementary) central elements in a variety with DFC and show that the coordinates (in a direct product representation) of a central element are central elements. Several results obtained in the previous sections are compiled in Section 5 to finish the proof of the Main Theorem. Two examples are treated in Section 6. In $\$ 6.1$ we present a variety with $\overrightarrow{0} \& \overrightarrow{1}$ that does not have DFC; this shows that our definitions do not trivialize. In $₫ 6.2$ we give an optimal formula for the case of semilattices.

Throughout this paper the following notation will be used. For $A \in \mathcal{V}$ and $\vec{a}, \vec{b} \in A^{n}, \mathrm{Cg}^{A}(\vec{a}, \vec{b})$ will denote the congruence generated by the set $\left\{\left(a_{k}, b_{k}\right): 1 \leq\right.$ $k \leq n\}$. The symbols $\nabla$ and $\Delta$ will stand for the universal and trivial congruences, respectively. We will use $\theta \times \theta^{*}=\Delta$ in place of " $\theta$ and $\theta^{*}$ are complementary factor 
congruences". The term algebra (in the language of $\mathcal{V}$ ) and the $\mathcal{V}$-free algebra on $X$ will be denoted by $T(X)$ and $F(X)$, respectively. The $i$-th component of an element $a$ in a direct product $\Pi_{i} A_{i}$ will be called $a^{i}=\pi_{i}(a)$; hence, if $a \in A_{0} \times A_{1}$, $a=\left(a^{0}, a^{1}\right)$. If elements $a, b$ of an algebra $A$ are related by a congruence $\theta \in \operatorname{Con} A$, we will write interchangeably $(a, b) \in \theta, a \theta b$ or $a \stackrel{\theta}{\equiv} b$. This notation generalizes to tuples, viz., $\vec{a} \theta \vec{b}$ means $\left(a_{i}, b_{i}\right) \in \theta$ for all $i$.

\section{The Left Determining Property: A Mal'Cev condition}

We will use the following Left Determining Property:

For every $A \in \mathcal{V}, \vec{e} \in A^{l}$ and $\varphi, \varphi^{*}, \theta, \theta^{*} \in \operatorname{Con} A$, if $\varphi \times \varphi^{*}=\Delta$, $\theta \times \theta^{*}=\Delta, \overrightarrow{0} \theta \vec{e} \theta^{*} \overrightarrow{1}$ and $\overrightarrow{0} \varphi \vec{e} \varphi^{*} \overrightarrow{1}$, then $\theta=\varphi$.

It is not difficult to see the implications: Determining Property $\Rightarrow$ Left Determining Property $\Rightarrow$ Weak Determining Property.

The following theorem gives a Mal'cev condition for the Left Determining Property. Let $s_{i}, t_{i}$ be $(2 i+l)$-ary terms (in the language of $\mathcal{V}$ ) for each $i=1, \ldots, n$ and let $A$ be an algebra in the language of $\mathcal{V}$ (not necessarily in $\mathcal{V}$ ). For $\left(c, d, \vec{e}, a_{1}, b_{1}, \ldots\right.$, $\left.a_{n}, b_{n}\right) \in A^{2+l+2 n}$, we define $\sigma\left(c, d, \vec{e}, a_{1}, b_{1}, \ldots, a_{n}, b_{n}\right)$ to be the tuple $\left(x, y, \vec{z}, x_{1}\right.$, $\left.y_{1}, \ldots, x_{n}, y_{n}\right)$ given by the following recursion:

$$
\begin{array}{ll}
x:=c & x_{j}:=s_{j}\left(x, y, \vec{z}, x_{1}, y_{1}, \ldots, x_{j-1}, y_{j-1}\right), \\
y:=c & y_{j}:=b_{j}, \\
\vec{z}:=\overrightarrow{0} . &
\end{array}
$$

We define $\sigma^{*}, \rho, \rho^{*}$ analogously.

- $\sigma^{*}\left(c, d, \vec{e}, a_{1}, b_{1}, \ldots, a_{n}, b_{n}\right):=\left(x, y, \vec{z}, x_{1}, y_{1}, \ldots, x_{n}, y_{n}\right)$ where:

$$
\begin{array}{ll}
x:=c & x_{j}:=t_{j}\left(x, y, \vec{z}, x_{1}, y_{1}, \ldots, x_{j-1}, y_{j-1}\right), \\
y:=d & y_{j}:=b_{j}, \\
\vec{z}:=\overrightarrow{1} . &
\end{array}
$$

- $\rho\left(c, d, \vec{e}, a_{1}, b_{1}, \ldots, a_{n}, b_{n}\right):=\left(x, y, \vec{z}, x_{1}, y_{1}, \ldots, x_{n}, y_{n}\right)$ where:

$$
\begin{array}{ll}
x:=c & x_{j}:=a_{j}, \\
y:=d & y_{j}:=s_{j}\left(x, y, \vec{z}, x_{1}, y_{1}, \ldots, x_{j-1}, y_{j-1}\right), \\
\vec{z}:=\overrightarrow{0} . &
\end{array}
$$

- $\rho^{*}\left(c, d, \vec{e}, a_{1}, b_{1}, \ldots, a_{n}, b_{n}\right):=\left(x, y, \vec{z}, x_{1}, y_{1}, \ldots, x_{n}, y_{n}\right)$ where:

$$
\begin{array}{ll}
x:=c & x_{j}:=a_{j}, \\
y:=d & y_{j}:=t_{j}\left(x, y, \vec{z}, x_{1}, y_{1}, \ldots, x_{j-1}, y_{j-1}\right), \\
\vec{z}:=\overrightarrow{1} . &
\end{array}
$$

We first state without proof a lemma concerning these functions. 
Lemma 2.1. For every $c, d, \vec{e}, a_{1}, b_{1}, \ldots, a_{n}, b_{n} \in A$, we have the following identities:

$$
\begin{array}{r}
\operatorname{Cg}(c, d) \vee \operatorname{Cg}(\vec{e}, \overrightarrow{0}) \vee \bigvee_{i} \operatorname{Cg}\left(a_{i}, s_{i}\left(c, d, \vec{e}, a_{1}, b_{1}, \ldots, a_{i-1}, b_{i-1}\right)\right) \\
=\operatorname{Cg}\left(\left(c, d, \vec{e}, a_{1}, b_{1}, \ldots, a_{n}, b_{n}\right), \sigma\left(c, d, \vec{e}, a_{1}, b_{1}, \ldots, a_{n}, b_{n}\right)\right), \\
\operatorname{Cg}(\vec{e}, \overrightarrow{1}) \vee \bigvee_{i} \operatorname{Cg}\left(a_{i}, t_{i}\left(c, d, \vec{e}, \ldots, a_{i-1}, b_{i-1}\right)\right) \\
=\operatorname{Cg}\left(\left(c, d, \vec{e}, \ldots, a_{n}, b_{n}\right), \sigma^{*}\left(c, d, \vec{e}, \ldots, a_{n}, b_{n}\right)\right), \\
\operatorname{Cg}(\vec{e}, \overrightarrow{0}) \vee \bigvee_{i} \operatorname{Cg}\left(b_{i}, s_{i}\left(c, d, \vec{e}, \ldots, a_{i-1}, b_{i-1}\right)\right) \\
=\operatorname{Cg}\left(\left(c, d, \vec{e}, \ldots, a_{n}, b_{n}\right), \rho\left(c, d, \vec{e}, \ldots, a_{n}, b_{n}\right)\right), \\
\operatorname{Cg}(\vec{e}, \overrightarrow{1}) \vee \bigvee_{i} \operatorname{Cg}\left(b_{i}, t_{i}\left(c, d, \vec{e}, \ldots, a_{i-1}, b_{i-1}\right)\right) \\
=\operatorname{Cg}\left(\left(c, d, \vec{e}, \ldots, a_{n}, b_{n}\right), \rho^{*}\left(c, d, \vec{e}, \ldots, a_{n}, b_{n}\right)\right) .
\end{array}
$$

In the proofs that follow, we will repeatedly find elements in an algebra that solve congruential "equations" of the form

$$
a \stackrel{\theta}{\equiv} x \stackrel{\theta^{*}}{\equiv} b
$$

when $\theta \times \theta^{*}=\Delta$. Using the functions $\sigma, \sigma^{*}, \rho$ and $\rho^{*}$ just defined, we can assert conclusions from the way elements such as $x$ are constructed. This is the content of the following immediate consequences of Lemma 2.1.

Corollary 2.2. Given $c, d, \vec{e} \in A$ and $\theta, \theta^{*} \in \operatorname{Con} A$ such that $\overrightarrow{0} \theta \vec{e} \theta^{*} \overrightarrow{1}$ and $c \theta d$, for every $a_{i}$ and $b_{i}$ with $i=1, \ldots, n$ such that

$$
\begin{aligned}
& s_{1}(c, d, \vec{e}) \stackrel{\theta}{\equiv} a_{1} \stackrel{\theta^{*}}{\equiv} t_{1}(c, d, \vec{e}), \\
& s_{2}\left(c, d, \vec{e}, a_{1}, b_{1}\right) \stackrel{\theta}{\equiv} a_{2} \stackrel{\theta^{*}}{\equiv} t_{2}\left(c, d, \vec{e}, a_{1}, b_{1}\right), \\
& s_{i+1}\left(c, d, \vec{e}, a_{1}, b_{1}, \ldots, a_{i}, b_{i}\right) \stackrel{\theta}{\equiv} a_{i+1} \stackrel{\theta^{*}}{\equiv} t_{i+1}\left(c, d, \vec{e}, a_{1}, b_{1}, \ldots, a_{i}, b_{i}\right),
\end{aligned}
$$

we have

$$
\begin{aligned}
& t\left(c, d, \vec{e}, a_{1}, b_{1}, \ldots, a_{n}, b_{n}\right) \stackrel{\theta}{\equiv} t\left(\sigma\left(c, d, \vec{e}, a_{1}, b_{1}, \ldots, a_{n}, b_{n}\right)\right), \\
& t\left(c, d, \vec{e}, a_{1}, b_{1}, \ldots, a_{n}, b_{n}\right) \stackrel{\theta^{*}}{\equiv} t\left(\sigma^{*}\left(c, d, \vec{e}, a_{1}, b_{1}, \ldots, a_{n}, b_{n}\right)\right),
\end{aligned}
$$

for every $(2 n+l+2)$-ary term $t$ in the language of $\mathcal{V}$.

The next result is entirely analogous.

Corollary 2.3. Suppose $c, d, \vec{e} \in A$ and $\varphi, \varphi^{*} \in \operatorname{Con} A$ such that $\overrightarrow{0} \varphi \vec{e} \varphi^{*} \overrightarrow{1}$. If $a_{i}$ and $b_{i}$ satisfy

$$
\begin{gathered}
s_{1}(c, d, \vec{e}) \stackrel{\varphi}{=} b_{1} \stackrel{\varphi^{*}}{=} t_{1}(c, d, \vec{e}), \\
\ldots \\
s_{i+1}\left(c, d, \vec{e}, a_{1}, b_{1}, \ldots, a_{i}, b_{i}\right) \stackrel{\varphi}{=} b_{i+1} \stackrel{\stackrel{\varphi^{*}}{=}}{=} t_{i+1}\left(c, d, \vec{e}, a_{1}, b_{1}, \ldots, a_{i}, b_{i}\right),
\end{gathered}
$$


we obtain

$$
\begin{aligned}
& t\left(c, d, \vec{e}, a_{1}, b_{1}, \ldots, a_{n}, b_{n}\right) \stackrel{\varphi}{=} t\left(\rho\left(c, d, \vec{e}, a_{1}, b_{1}, \ldots, a_{n}, b_{n}\right)\right), \\
& t\left(c, d, \vec{e}, a_{1}, b_{1}, \ldots, a_{n}, b_{n}\right) \stackrel{\varphi^{*}}{=} t\left(\rho^{*}\left(c, d, \vec{e}, a_{1}, b_{1}, \ldots, a_{n}, b_{n}\right)\right)
\end{aligned}
$$

for every $(2 n+l+2)$-ary term $t$ in the language of $\mathcal{V}$.

We will also need the following (Grätzer's) version of Mal'cev's key observation on principal congruences.

Lemma 2.4. Let $A$ be any algebra and let $a, b \in A, \vec{a}, \vec{b} \in A^{n}$. Then $(a, b) \in$ $\mathrm{Cg}^{A}(\vec{a}, \vec{b})$ if and only if there exist $(n+m)$-ary terms $p_{1}(\vec{x}, \vec{u}), \ldots, p_{k}(\vec{x}, \vec{u})$, with $k$ odd, and $\vec{u} \in A^{m}$ such that:

$$
\begin{aligned}
a & =p_{1}(\vec{a}, \vec{u}), \\
p_{i}(\vec{b}, \vec{u}) & =p_{i+1}(\vec{b}, \vec{u}), i \text { odd } \\
p_{i}(\vec{a}, \vec{u}) & =p_{i+1}(\vec{a}, \vec{u}), i \text { even }, \\
p_{k}(\vec{b}, \vec{u}) & =b
\end{aligned}
$$

We will use $|\alpha|$ to denote the length of a word $\alpha$ and $\varepsilon$ will denote the empty word.

Theorem 2.5. Let $\mathcal{V}$ be a variety with $\overrightarrow{0} \& \overrightarrow{1}$. $\mathcal{V}$ has Left Determining Property if and only if there exist integers $N=2 k$ and $n,(2 i+l)$-ary terms $s_{i}$ and $t_{i}$ for each $i=1, \ldots, n$, and for every word $\alpha$ in the alphabet $\{1, \ldots, N\}$ of length no greater than $N$ there are terms $L_{\alpha}, R_{\alpha}$ such that

$$
|\alpha|=N
$$

$$
\begin{aligned}
L_{\alpha}(\rho(\vec{X})) & \approx R_{\alpha}(\rho(\vec{X})), \\
L_{\alpha}\left(\rho^{*}(\vec{X})\right) & \approx R_{\alpha}\left(\rho^{*}(\vec{X})\right),
\end{aligned}
$$

$|\alpha|=0$

$$
\begin{aligned}
x & \approx L_{\varepsilon}\left(x, y, \vec{z}, x_{1}, y_{1}, \ldots, x_{n}, y_{n}\right), \\
& R_{\varepsilon}\left(x, y, \vec{z}, x_{1}, y_{1}, \ldots, x_{n}, y_{n}\right) \approx y, \\
L_{\varepsilon}(\rho(\vec{X})) & \approx L_{1}(\rho(\vec{X})), \\
R_{j}(\rho(\vec{X})) & \approx L_{j+1}(\rho(\vec{X})) \quad \text { if } 1 \leq j \leq N-1, \\
R_{N}(\rho(\vec{X})) & \approx R_{\varepsilon}(\rho(\vec{X})) .
\end{aligned}
$$


$0<|\alpha|<N$

If $|\alpha|$ is even, then

$$
\begin{aligned}
L_{\alpha}(\rho(\vec{X})) & \approx L_{\alpha 1}(\rho(\vec{X})), \\
R_{\alpha j}(\rho(\vec{X})) & \approx L_{\alpha(j+1)}(\rho(\vec{X})) \quad \text { if } 1 \leq j \leq k-1, \\
R_{\alpha k}(\rho(\vec{X})) & \approx R_{\alpha}(\rho(\vec{X})), \\
L_{\alpha}\left(\rho^{*}(\vec{X})\right) & \approx L_{\alpha(k+1)}\left(\rho^{*}(\vec{X})\right), \\
R_{\alpha j}\left(\rho^{*}(\vec{X})\right) & \approx L_{\alpha(j+1)}\left(\rho^{*}(\vec{X})\right) \quad \text { if } k+1 \leq j \leq N-1, \\
R_{\alpha N}\left(\rho^{*}(\vec{X})\right) & \approx R_{\alpha}\left(\rho^{*}(\vec{X})\right) .
\end{aligned}
$$

If $|\alpha|$ is odd, then

$$
\begin{aligned}
L_{\alpha}(\sigma(\vec{X})) & \approx L_{\alpha 1}(\sigma(\vec{X})), \\
R_{\alpha j}(\sigma(\vec{X})) & \approx L_{\alpha(j+1)}(\sigma(\vec{X})) \quad \text { if } 1 \leq j \leq k-1, \\
R_{\alpha k}(\sigma(\vec{X})) & \approx R_{\alpha}(\sigma(\vec{X})), \\
L_{\alpha}\left(\sigma^{*}(\vec{X})\right) & \approx L_{\alpha(k+1)}\left(\sigma^{*}(\vec{X})\right), \\
R_{\alpha j}\left(\sigma^{*}(\vec{X})\right) & \approx L_{\alpha(j+1)}\left(\sigma^{*}(\vec{X})\right) \quad \text { if } k+1 \leq j \leq N-1, \\
R_{\alpha N}\left(\sigma^{*}(\vec{X})\right) & \approx R_{\alpha}\left(\sigma^{*}(\vec{X})\right),
\end{aligned}
$$

where $\vec{X}=\left(x, y, \vec{z}, x_{1}, y_{1}, \ldots, x_{n}, y_{n}\right)$ and $\sigma, \sigma^{*}, \rho$ and $\rho^{*}$ are defined relative to $s_{i}$, $t_{i}$, on $T(\vec{X})$.

Proof. $(\Leftarrow)$ Assume the existence of the terms, and suppose $\varphi \times \varphi^{*}=\Delta, \theta \times \theta^{*}=\Delta$, $\overrightarrow{0} \theta e \theta^{*} \overrightarrow{1}, \overrightarrow{0} \varphi e \varphi^{*} \overrightarrow{1}$, and $c \theta d$. We want to see $c \varphi d$. There exist unique $a_{i}, b_{i}$ satisfying the following relations:

$$
\begin{gathered}
s_{1}(c, d, \vec{e}) \stackrel{\ominus}{\equiv} a_{1} \stackrel{\theta^{*}}{\equiv} t_{1}(c, d, \vec{e}), \\
s_{1}(c, d, \vec{e}) \stackrel{\stackrel{\varphi}{\equiv}}{=} b_{1} \stackrel{\varphi^{*}}{\equiv} t_{1}(c, d, \vec{e}), \\
\ldots \\
s_{j+1}\left(c, d, \vec{e}, a_{1}, b_{1}, \ldots, a_{j}, b_{j}\right) \stackrel{\ominus}{\equiv} a_{j+1} \stackrel{\theta^{*}}{\equiv} t_{j+1}\left(c, d, \vec{e}, a_{1}, b_{1}, \ldots, a_{j}, b_{j}\right), \\
s_{j+1}\left(c, d, \vec{e}, a_{1}, b_{1}, \ldots, a_{j}, b_{j}\right) \stackrel{\varphi}{=} b_{j+1} \stackrel{\underline{\varphi}^{*}}{=} t_{j+1}\left(c, d, \vec{e}, a_{1}, b_{1}, \ldots, a_{j}, b_{j}\right) .
\end{gathered}
$$

Note that their definition combines the schemes in Corollaries 2.2 and 2.3 So, by equations (2.2) and (2.3) we have, taking $t:=L_{\alpha}, R_{\alpha}$ :

$L_{\alpha}\left(\sigma\left(c, d, \vec{e}, a_{1}, b_{1}, \ldots\right)\right) \stackrel{\theta}{\equiv} L_{\alpha}\left(c, d, \vec{e}, a_{1}, b_{1}, \ldots, a_{n}, b_{n}\right) \stackrel{\theta^{*}}{\equiv} L_{\alpha}\left(\sigma^{*}\left(c, d, \vec{e}, a_{1}, b_{1}, \ldots\right)\right)$, $L_{\alpha}\left(\rho\left(c, d, \vec{e}, a_{1}, b_{1}, \ldots\right)\right) \stackrel{\varphi}{\equiv} L_{\alpha}\left(c, d, \vec{e}, a_{1}, b_{1}, \ldots, a_{n}, b_{n}\right) \stackrel{\varphi^{*}}{\equiv} L_{\alpha}\left(\rho^{*}\left(c, d, \vec{e}, a_{1}, b_{1}, \ldots\right)\right)$,

$$
\begin{aligned}
& R_{\alpha}\left(\sigma\left(c, d, \vec{e}, a_{1}, b_{1}, \ldots\right)\right) \stackrel{\ominus}{\equiv} R_{\alpha}\left(c, d, \vec{e}, a_{1}, b_{1}, \ldots, a_{n}, b_{n}\right) \stackrel{\theta^{*}}{\equiv} R_{\alpha}\left(\sigma^{*}\left(c, d, \vec{e}, a_{1}, b_{1}, \ldots\right)\right), \\
& R_{\alpha}\left(\rho\left(c, d, \vec{e}, a_{1}, b_{1}, \ldots\right)\right) \stackrel{\varphi}{\equiv} R_{\alpha}\left(c, d, \vec{e}, a_{1}, b_{1}, \ldots, a_{n}, b_{n}\right) \stackrel{\varphi^{*}}{=} R_{\alpha}\left(\rho^{*}\left(c, d, \vec{e}, a_{1}, b_{1}, \ldots\right)\right)
\end{aligned}
$$


for every $\alpha$. We'll prove inductively that

$$
L_{\alpha}\left(c, d, \vec{e}, a_{1}, b_{1}, \ldots, a_{n}, b_{n}\right)=R_{\alpha}\left(c, d, \vec{e}, a_{1}, b_{1}, \ldots, a_{n}, b_{n}\right)
$$

for all $\alpha \neq \varepsilon$. Take $\alpha$ such that $|\alpha|=N$. Then

$$
\begin{aligned}
L_{\alpha}\left(c, d, \vec{e}, a_{1}, b_{1}, \ldots, a_{n}, b_{n}\right) & \stackrel{\varphi}{=} L_{\alpha}\left(\rho\left(c, d, \vec{e}, a_{1}, b_{1}, \ldots, a_{n}, b_{n}\right)\right) & & \text { by (2.15) } \\
& =R_{\alpha}\left(\rho\left(c, d, \vec{e}, a_{1}, b_{1}, \ldots, a_{n}, b_{n}\right)\right) & & \text { using (2.4) } \\
& \stackrel{\varphi}{=} R_{\alpha}\left(c, d, \vec{e}, a_{1}, b_{1}, \ldots, a_{n}, b_{n}\right) & & \text { by (2.16) }
\end{aligned}
$$

and

$$
\begin{aligned}
& L_{\alpha}\left(c, d, \vec{e}, a_{1}, b_{1}, \ldots, a_{n}, b_{n}\right) \stackrel{\varphi^{*}}{=} L_{\alpha}\left(\rho^{*}\left(c, d, \vec{e}, a_{1}, b_{1}, \ldots, a_{n}, b_{n}\right)\right) \text { by 2.15) } \\
& =R_{\alpha}\left(\rho^{*}\left(c, d, \vec{e}, a_{1}, b_{1}, \ldots, a_{n}, b_{n}\right)\right) \quad \text { using (2.4) } \\
& \stackrel{\varphi^{*}}{=} R_{\alpha}\left(c, d, \vec{e}, a_{1}, b_{1}, \ldots, a_{n}, b_{n}\right) \quad \text { by (2.16). }
\end{aligned}
$$

Hence $\left(L_{\alpha}\left(c, d, \vec{e}, a_{1}, b_{1}, \ldots, a_{n}, b_{n}\right), R_{\alpha}\left(c, d, \vec{e}, a_{1}, b_{1}, \ldots, a_{n}, b_{n}\right)\right) \in \varphi \cap \varphi^{*}=\Delta$ and then $L_{\alpha}\left(c, d, \vec{e}, a_{1}, b_{1}, \ldots, a_{n}, b_{n}\right)=R_{\alpha}\left(c, d, \vec{e}, a_{1}, b_{1}, \ldots, a_{n}, b_{n}\right)$.

Take $\alpha \neq \varepsilon$ of odd length and assume

$$
L_{\alpha j}\left(c, d, \vec{e}, a_{1}, b_{1}, \ldots, a_{n}, b_{n}\right)=R_{\alpha j}\left(c, d, \vec{e}, a_{1}, b_{1}, \ldots, a_{n}, b_{n}\right)
$$

for every $j=1, \ldots, N$. We check that

$$
L_{\alpha}\left(c, d, \vec{e}, a_{1}, b_{1}, \ldots, a_{n}, b_{n}\right) \stackrel{\theta}{\equiv} R_{\alpha}\left(c, d, \vec{e}, a_{1}, b_{1}, \ldots, a_{n}, b_{n}\right),
$$

$$
\begin{aligned}
& L_{\alpha}\left(c, d, \vec{e}, a_{1}, b_{1}, \ldots, a_{n}, b_{n}\right) \stackrel{\theta}{=} L_{\alpha}\left(\sigma\left(c, d, \vec{e}, a_{1}, b_{1}, \ldots, a_{n}, b_{n}\right)\right) \quad \text { by (2.15) } \\
& =L_{\alpha 1}\left(\sigma\left(c, d, \vec{e}, a_{1}, b_{1}, \ldots, a_{n}, b_{n}\right)\right) \quad \text { by (2.13) } \\
& \stackrel{\theta}{\equiv} L_{\alpha 1}\left(c, d, \vec{e}, a_{1}, b_{1}, \ldots, a_{n}, b_{n}\right) \quad \text { by }(2.15) \\
& =R_{\alpha 1}\left(c, d, \vec{e}, a_{1}, b_{1}, \ldots, a_{n}, b_{n}\right) \quad \text { by ind. hypothesis } \\
& \stackrel{\theta}{\equiv} R_{\alpha 1}\left(\sigma\left(c, d, \vec{e}, a_{1}, b_{1}, \ldots, a_{n}, b_{n}\right)\right) \quad \text { by (2.16) } \\
& \stackrel{\theta}{\equiv} \ldots \quad \text { using (2.13) . . } \\
& =R_{\alpha k}\left(\sigma\left(c, d, \vec{e}, a_{1}, b_{1}, \ldots, a_{n}, b_{n}\right)\right) \\
& =R_{\alpha}\left(\sigma\left(c, d, \vec{e}, a_{1}, b_{1}, \ldots, a_{n}, b_{n}\right)\right) \quad \text { using (2.13) } \\
& \stackrel{\ominus}{\equiv} R_{\alpha}\left(c, d, \vec{e}, a_{1}, b_{1}, \ldots, a_{n}, b_{n}\right) \text {. }
\end{aligned}
$$


In the same way for $\theta^{*}$, we have

$$
\begin{array}{rlrl}
L_{\alpha}\left(c, d, \vec{e}, a_{1}, b_{1}, \ldots, a_{n}, b_{n}\right) & \stackrel{\theta^{*}}{\equiv} L_{\alpha}\left(\sigma^{*}\left(c, d, \vec{e}, a_{1}, b_{1}, \ldots, a_{n}, b_{n}\right)\right) & \text { by (2.15) } \\
& =L_{\alpha(k+1)}\left(\sigma^{*}\left(c, d, \vec{e}, a_{1}, b_{1}, \ldots, a_{n}, b_{n}\right)\right) & & \text { by (2.14) } \\
& \stackrel{\theta^{*}}{\equiv} L_{\alpha(k+1)}\left(c, d, \vec{e}, a_{1}, b_{1}, \ldots, a_{n}, b_{n}\right) & & \text { by (2.15) } \\
& =R_{\alpha(k+1)}\left(c, d, \vec{e}, a_{1}, b_{1}, \ldots, a_{n}, b_{n}\right) & & \text { by ind. hyp. } \\
& \stackrel{\theta^{*}}{\equiv} R_{\alpha(k+1)}\left(\sigma^{*}\left(c, d, \vec{e}, a_{1}, b_{1}, \ldots, a_{n}, b_{n}\right)\right) & & \text { by (2.16) } \\
& \stackrel{\theta^{*}}{\equiv} \ldots & \text { using (2.14) } \ldots \\
& =R_{\alpha N}\left(\sigma^{*}\left(c, d, \vec{e}, a_{1}, b_{1}, \ldots, a_{n}, b_{n}\right)\right) & \\
& =R_{\alpha}\left(\sigma^{*}\left(c, d, \vec{e}, a_{1}, b_{1}, \ldots, a_{n}, b_{n}\right)\right) & & \text { using (2.14) } \\
& \stackrel{\theta^{*}}{\equiv} R_{\alpha}\left(c, d, \vec{e}, a_{1}, b_{1}, \ldots, a_{n}, b_{n}\right) & & \text { by (2.16). }
\end{array}
$$

Hence $\left(L_{\alpha}\left(c, d, \vec{e}, a_{1}, b_{1}, \ldots, a_{n}, b_{n}\right), R_{\alpha}\left(c, d, \vec{e}, a_{1}, b_{1}, \ldots, a_{n}, b_{n}\right)\right) \in \theta \cap \theta^{*}=\Delta$, and therefore they are equal.

If $\alpha \neq \varepsilon$ has even length, then

$$
\begin{aligned}
& L_{\alpha}\left(c, d, \vec{e}, a_{1}, b_{1}, \ldots, a_{n}, b_{n}\right) \stackrel{\varphi}{=} L_{\alpha}\left(\rho\left(c, d, \vec{e}, a_{1}, b_{1}, \ldots, a_{n}, b_{n}\right)\right) \quad \text { by (2.15) } \\
& =L_{\alpha 1}\left(\rho\left(c, d, \vec{e}, a_{1}, b_{1}, \ldots, a_{n}, b_{n}\right)\right) \quad \text { by (2.9) } \\
& \stackrel{\varphi}{=} L_{\alpha 1}\left(c, d, \vec{e}, a_{1}, b_{1}, \ldots, a_{n}, b_{n}\right) \quad \text { by (2.15) } \\
& =R_{\alpha 1}\left(c, d, \vec{e}, a_{1}, b_{1}, \ldots, a_{n}, b_{n}\right) \quad \text { by ind. hypothesis } \\
& \stackrel{\varphi}{\equiv} R_{\alpha 1}\left(\rho\left(c, d, \vec{e}, a_{1}, b_{1}, \ldots, a_{n}, b_{n}\right)\right) \text { by (2.16) } \\
& \underline{\underline{\underline{\varphi}}} \ldots \quad \text { using (2.10) } \ldots \\
& =R_{\alpha k}\left(\rho\left(c, d, \vec{e}, a_{1}, b_{1}, \ldots, a_{n}, b_{n}\right)\right) \\
& =R_{\alpha}\left(\rho\left(c, d, \vec{e}, a_{1}, b_{1}, \ldots, a_{n}, b_{n}\right)\right) \quad \text { using (2.11) } \\
& \stackrel{\varphi}{\equiv} R_{\alpha}\left(c, d, \vec{e}, a_{1}, b_{1}, \ldots, a_{n}, b_{n}\right) \quad \text { by (2.16) }
\end{aligned}
$$

proves $\left(L_{\alpha}\left(c, d, \vec{e}, a_{1}, b_{1}, \ldots, a_{n}, b_{n}\right), R_{\alpha}\left(c, d, \vec{e}, a_{1}, b_{1}, \ldots, a_{n}, b_{n}\right)\right) \in \varphi$, and

$$
\begin{array}{rlrl}
L_{\alpha}\left(c, d, \vec{e}, a_{1}, b_{1}, \ldots, a_{n}, b_{n}\right) & \stackrel{\varphi^{*}}{\equiv} L_{\alpha}\left(\rho^{*}\left(c, d, \vec{e}, a_{1}, b_{1}, \ldots, a_{n}, b_{n}\right)\right) & & \text { by (2.15) } \\
& =L_{\alpha(k+1)}\left(\rho^{*}\left(c, d, \vec{e}, a_{1}, b_{1}, \ldots, a_{n}, b_{n}\right)\right) & & \text { by (2.12) } \\
& \stackrel{\varphi^{*}}{=} L_{\alpha(k+1)}\left(c, d, \vec{e}, a_{1}, b_{1}, \ldots, a_{n}, b_{n}\right) & & \text { by (2.15) } \\
& =R_{\alpha(k+1)}\left(c, d, \vec{e}, a_{1}, b_{1}, \ldots, a_{n}, b_{n}\right) & & \text { by ind. hyp. } \\
& \stackrel{\varphi^{*}}{=} R_{\alpha(k+1)}\left(\rho^{*}\left(c, d, \vec{e}, a_{1}, b_{1}, \ldots, a_{n}, b_{n}\right)\right) & & \text { by (2.16) } \\
& \stackrel{\varphi^{*}}{=} \ldots & & \text { using (2.12). } \\
& =R_{\alpha N}\left(\rho^{*}\left(c, d, \vec{e}, a_{1}, b_{1}, \ldots, a_{n}, b_{n}\right)\right) & \\
& =R_{\alpha}\left(\rho^{*}\left(c, d, \vec{e}, a_{1}, b_{1}, \ldots, a_{n}, b_{n}\right)\right) & & \text { using (2.12) } \\
& \stackrel{\varphi^{*}}{=} R_{\alpha}\left(c, d, \vec{e}, a_{1}, b_{1}, \ldots, a_{n}, b_{n}\right) & & \text { by (2.16) }
\end{array}
$$


completes this case. Finally, we have:

$$
\begin{aligned}
& c=L_{\varepsilon}\left(\rho\left(c, d, \vec{e}, a_{1}, b_{1}, \ldots, a_{n}, b_{n}\right)\right) \quad \text { using (2.5) } \\
& =L_{1}\left(\rho\left(c, d, \vec{e}, a_{1}, b_{1}, \ldots, a_{n}, b_{n}\right)\right) \quad \text { by (2.6) } \\
& \stackrel{\varphi}{=} L_{1}\left(c, d, \vec{e}, a_{1}, b_{1}, \ldots, a_{n}, b_{n}\right) \quad \text { by (2.15) } \\
& =R_{1}\left(c, d, \vec{e}, a_{1}, b_{1}, \ldots, a_{n}, b_{n}\right) \quad \text { by (2.17) } \\
& \stackrel{\varphi}{=} R_{1}\left(\rho\left(c, d, \vec{e}, a_{1}, b_{1}, \ldots, a_{n}, b_{n}\right)\right) \quad \text { by (2.16) } \\
& \underline{\underline{\underline{\varphi}}} \ldots \quad \text { using (2.7) } \ldots \\
& =R_{N}\left(\rho\left(c, d, \vec{e}, a_{1}, b_{1}, \ldots, a_{n}, b_{n}\right)\right) \\
& =R_{\varepsilon}\left(\rho\left(c, d, \vec{e}, a_{1}, b_{1}, \ldots, a_{n}, b_{n}\right)\right) \quad \text { using (2.8) } \\
& =d \quad \text { using (2.5). }
\end{aligned}
$$

This proves $(c, d) \in \varphi$.

$(\Rightarrow)$ For each set of variables $Y$, define

$$
\begin{aligned}
Y^{*} & :=Y \cup\left\{x_{p, q}: p, q \in T(Y)\right\} \cup\left\{y_{p, q}: p, q \in T(Y)\right\}, \\
Y^{0 *} & :=Y, \\
Y^{(n+1) *} & :=\left(Y^{n *}\right)^{*}, \\
Y^{\infty} & :=\bigcup_{n \geq 1} Y^{n *},
\end{aligned}
$$

where $x_{p, q}$ and $y_{p, q}$ are new variables. Take $Z:=\left\{x, y, z_{1}, \ldots, z_{l}\right\}$ and $F:=F\left(Z^{\infty}\right)$. Define the index of $p \in T\left(Z^{\infty}\right)$ as $\operatorname{ind}(p)=\min \left\{j: p \in T\left(Z^{j *}\right)\right\}$; it is evident that if $\operatorname{ind}\left(x_{p, q}\right) \leq \operatorname{ind}\left(x_{r, s}\right)$, neither $p$ nor $q$ can be terms depending on $x_{r, s}$. The same holds for $\operatorname{ind}\left(x_{p, q}\right) \leq \operatorname{ind}\left(y_{r, s}\right)$ and symmetrically, and for $\operatorname{ind}\left(y_{p, q}\right) \leq \operatorname{ind}\left(y_{r, s}\right)$.

Take the following congruences on $F$ :

$$
\begin{aligned}
& \theta:=\operatorname{Cg}(\overrightarrow{0}, \vec{z}) \vee \operatorname{Cg}(x, y) \vee \bigvee\left\{\operatorname{Cg}\left(p, x_{p, q}\right): p, q \in F\right\}, \quad \delta_{0}=\epsilon_{0}:=\Delta^{F}, \\
& \theta^{*}:=\operatorname{Cg}(\overrightarrow{1}, \vec{z}) \vee \bigvee\left\{\operatorname{Cg}\left(x_{p, q}, q\right): p, q \in F\right\}, \quad \delta_{n+1}:=\left(\theta \vee \epsilon_{n}\right) \cap\left(\theta^{*} \vee \epsilon_{n}\right), \\
& \varphi:=\operatorname{Cg}(\overrightarrow{0}, \vec{z}) \vee \bigvee\left\{\operatorname{Cg}\left(p, y_{p, q}\right): p, q \in F\right\}, \quad \epsilon_{n+1}:=\left(\varphi \vee \delta_{n}\right) \cap\left(\varphi^{*} \vee \delta_{n}\right), \\
& \varphi^{*}:=\operatorname{Cg}(\overrightarrow{1}, \vec{z}) \vee \bigvee\left\{\operatorname{Cg}\left(y_{p, q}, q\right): p, q \in F\right\}, \quad \delta_{\infty}:=\bigvee_{n \geq 0} \delta_{n}=\bigvee_{n \geq 0} \epsilon_{n} .
\end{aligned}
$$

By construction, $\varphi \circ \varphi^{*}=\theta \circ \theta^{*}=\nabla^{F}, \overrightarrow{0} \theta \vec{z} \theta^{*} \overrightarrow{1}, \overrightarrow{0} \varphi \vec{z} \varphi^{*} \overrightarrow{1}$, and $x \theta y$. Observe that if $(a, b) \in\left(\varphi \vee \delta_{\infty}\right) \cap\left(\varphi^{*} \vee \delta_{\infty}\right)$, then there exists an $n \geq 0$ such that $(a, b) \in$ $\left(\varphi \vee \delta_{n}\right) \cap\left(\varphi^{*} \vee \delta_{n}\right)$. But this congruence is exactly $\epsilon_{n+1}$; hence $(a, b) \in \epsilon_{n+1} \subseteq \delta_{\infty}$. We may conclude $\left(\varphi \vee \delta_{\infty}\right) \cap\left(\varphi^{*} \vee \delta_{\infty}\right)=\delta_{\infty}$. The same happens with $\theta$ and $\theta^{*}$; hence

$$
\left(\varphi \vee \delta_{\infty}\right) / \delta_{\infty} \times\left(\varphi^{*} \vee \delta_{\infty}\right) / \delta_{\infty}=\Delta, \quad\left(\theta \vee \delta_{\infty}\right) / \delta_{\infty} \times\left(\theta^{*} \vee \delta_{\infty}\right) / \delta_{\infty}=\Delta
$$

in $F / \delta_{\infty}$. Then, by the Left Determining Property we have $\left(x / \delta_{\infty}, y / \delta_{\infty}\right) \in$ $\left(\varphi \vee \delta_{\infty}\right) / \delta_{\infty}$ and hence $(x, y) \in \varphi \vee \delta_{\infty}$. We may find an even integer $N=2 k$ such that $(x, y) \in \varphi \circ^{2 N} \delta_{N}^{N}$, where $\delta_{N}^{N}$ is the result of replacing each occurrence of " $\mathrm{V}$ " in the definition of $\delta_{N}$ by $\circ^{N}$, the $n$-fold relational product. 
We will inductively define terms $L_{\alpha}$ and $R_{\alpha}$, for $\alpha$ a word of length at most $N$ in the alphabet $\{1, \ldots, N\}$ such that:

$$
\begin{array}{rlrl}
x & =L_{\varepsilon}, & & y=R_{\varepsilon}, \\
\left(L_{\varepsilon}, L_{1}\right) & \in \varphi, & & \left(R_{N}, R_{\varepsilon}\right) \in \varphi, \\
\left(L_{i}, R_{i}\right) & \in \delta_{N}^{N} & \text { if } 1 \leq i \leq N, & \\
\left(R_{i}, L_{(i+1)}\right) & \in \varphi \quad \text { if } 1 \leq i \leq N-1 . &
\end{array}
$$

For $\alpha \neq \varepsilon$ with $|\alpha|<N$ an odd integer,

$$
\begin{aligned}
\left(L_{\alpha}, L_{\alpha 1}\right) & \in \theta, & & \left(R_{\alpha k}, R_{\alpha}\right) \in \theta, \\
\left(L_{\alpha}, L_{\alpha(k+1)}\right) & \in \theta^{*}, & & \left(R_{\alpha N}, R_{\alpha}\right) \in \theta^{*}, \\
\left(L_{\alpha i}, R_{\alpha i}\right) & \in \epsilon_{N-|\alpha|}^{N} \quad \text { if } 1 \leq i \leq N, & & \\
\left(R_{\alpha i}, L_{\alpha(i+1)}\right) & \in \theta \quad \text { if } 1 \leq i \leq k-1, & & \\
\left(R_{\alpha i}, L_{\alpha(i+1)}\right) & \in \theta^{*} & \text { if } k+1 \leq i \leq N-1 &
\end{aligned}
$$

and for $\alpha \neq \varepsilon$ with $|\alpha|<N$ an even integer:

$$
\begin{array}{rlrl}
\left(L_{\alpha}, L_{\alpha 1}\right) & \in \varphi, & & \left(R_{\alpha k}, R_{\alpha}\right) \in \varphi, \\
\left(L_{\alpha}, L_{\alpha(k+1)}\right) & \in \varphi^{*}, & & \left(R_{\alpha N}, R_{\alpha}\right) \in \varphi^{*}, \\
\left(L_{\alpha i}, R_{\alpha i}\right) & \in \delta_{N-|\alpha|}^{N \mid} \quad \text { if } 1 \leq i \leq N, & \\
\left(R_{\alpha i}, L_{\alpha(i+1)}\right) & \in \varphi \quad \text { if } 1 \leq i \leq k-1, & \\
\left(R_{\alpha i}, L_{\alpha(i+1)}\right) & \in \varphi^{*} & \text { if } k+1 \leq i \leq N-1 . &
\end{array}
$$

We take $L_{\varepsilon}:=x$ and $R_{\varepsilon}:=y$. Since we know $(x, y) \in \varphi \circ^{2 N} \delta_{N}^{N}$, we define $L_{i}$, $R_{i}$ for $i=1, \ldots, N$ to be terms satisfying

$$
x \varphi L_{1} \delta_{N}^{N} R_{1} \varphi L_{2} \delta_{N}^{N} \cdots \varphi L_{N} \delta_{N}^{N} R_{N} \varphi y .
$$

Note that these terms satisfy (2.18)-(2.31) whenever they can be checked.

Suppose we have defined the terms corresponding to words with length less than or equal to $j$ and that they satisfy equations among (2.18)-(2.31) that involve words of length $j$ or shorter. Then we shall define terms corresponding to words with length equal to $j+1$ such that the totality of terms defined satisfy equations among (2.18) -2.31) which involve words of length $j+1$ or shorter. We have two cases:

Case 1: $j$ odd. Take $\alpha$, with $|\alpha|=j$. We have $L_{\alpha}$ and $R_{\alpha}$ and by (2.29), they satisfy $\left(L_{\alpha}, R_{\alpha}\right) \in \delta_{N-j+1}^{N}=\left(\theta \circ^{N} \epsilon_{N-j}^{N}\right) \cap\left(\theta^{*} \circ^{N} \epsilon_{N-j}^{N}\right)$. We define $L_{\alpha i}$ and $R_{\alpha i}$ for $i=1, \ldots, N$ such that:

$$
\begin{array}{r}
L_{\alpha} \theta L_{\alpha 1} \epsilon_{N-j}^{N} R_{\alpha 1} \theta L_{\alpha 2} \cdots R_{\alpha(k-1)} \theta L_{\alpha k} \epsilon_{N-j}^{N} R_{\alpha k} \theta R_{\alpha}, \\
L_{\alpha} \theta^{*} L_{\alpha(k+1)} \epsilon_{N-j}^{N} R_{\alpha(k+1)} \theta^{*} L_{\alpha(k+2)} \cdots L_{\alpha N} \epsilon_{N-j}^{N} R_{\alpha N} \theta^{*} R_{\alpha} .
\end{array}
$$

The equations among (2.18)-(2.31) which involve terms $L_{\mu}$ and $R_{\mu}$ with $|\mu|=j+1$ are (2.22) -(2.26). All of them can be inferred from (2.32).

Case 2: $j$ even. Take $\alpha$, with $|\alpha|=j$. We define $L_{\alpha i}$ and $R_{\alpha i}$ for $i=1, \ldots, N$. By (2.24) and by the definition of $\epsilon_{N-j+1}^{N}$ we may define our terms satisfying:

$$
\begin{array}{r}
L_{\alpha} \varphi L_{\alpha 1} \delta_{N-j}^{N} R_{\alpha 1} \varphi L_{\alpha 2} \cdots R_{\alpha(k-1)} \varphi L_{\alpha k} \delta_{N-j}^{N} R_{\alpha k} \varphi R_{\alpha}, \\
L_{\alpha} \varphi L_{\alpha(k+1)} \delta_{N-j}^{N} R_{\alpha(k+1)} \varphi^{*} L_{\alpha(k+2)} \cdots R_{\alpha N} \varphi^{*} R_{\alpha} .
\end{array}
$$


From this we immediately conclude (2.27)-2.31).

Let $V \subseteq Z^{\infty}$ be a finite set of variables such that if we replace $\theta, \theta^{*}, \varphi$ and $\varphi^{*}$, respectively, by the following compact congruences:

$$
\begin{aligned}
\theta_{0} & :=\operatorname{Cg}(\overrightarrow{0}, \vec{z}) \vee \operatorname{Cg}(x, y) \vee \bigvee\left\{\operatorname{Cg}\left(p, x_{p, q}\right): x_{p, q} \in V\right\}, \\
\theta_{0}^{*} & :=\operatorname{Cg}(\overrightarrow{1}, \vec{z}) \vee \bigvee\left\{\operatorname{Cg}\left(x_{p, q}, q\right): x_{p, q} \in V\right\}, \\
\varphi_{0} & :=\operatorname{Cg}(\overrightarrow{0}, \vec{z}) \vee \bigvee\left\{\operatorname{Cg}\left(p, y_{p, q}\right): y_{p, q} \in V\right\}, \\
\varphi^{*}{ }_{0} & :=\operatorname{Cg}(\overrightarrow{1}, \vec{z}) \vee \bigvee\left\{\operatorname{Cg}\left(y_{p, q}, q\right): y_{p, q} \in V\right\},
\end{aligned}
$$

then we still obtain congruential relations (2.18)-(2.31) (excepting (2.20), (2.29) and (2.24) $)$. It is clear that if we enlarge the set $V$ to a new set $X$, the properties enumerated will still hold.

Let $V_{0}$ be the union of $V$ and the (finite) set of variables occurring in terms $L_{\alpha}, R_{\alpha}$ with $\alpha$ a word. Define:

$$
V_{n+1}:=V_{n} \cup \bigcup\left\{\operatorname{Var}(p), \operatorname{Var}(q): x_{p, q} \in V_{n} \text { or } y_{p, q} \in V_{n}\right\}
$$

Hence, for some $M$ we have $V_{M}=V_{M+1}$; set

$$
X:=\left(V_{M} \cup\left\{x_{p, q}: y_{p, q} \in V_{M}\right\} \cup\left\{y_{p, q}: x_{p, q} \in V_{M}\right\}\right) \backslash\left\{x, y, z_{1}, \ldots, z_{l}\right\} .
$$

Order $X$ totally so that ind $: X \rightarrow \omega$ is nondecreasing and $x_{p, q}$ is the immediate predecessor of $y_{p, q}$, and add $x, y, z_{1}, \ldots, z_{l}$ at the beginning; we have

$$
\vec{X}=\left(x, y, \vec{z}, x_{s_{1}, t_{1}}, y_{s_{1}, t_{1}}, \ldots, x_{s_{n}, t_{n}}, y_{s_{n}, t_{n}}\right)=\left(x, y, \vec{z}, x_{1}, y_{1}, \ldots, x_{n}, y_{n}\right) .
$$

We may consider then $L_{\alpha}=L_{\alpha}(\vec{X})$ and the same for $R_{\alpha}$, and by the remarks after the definition of $Z^{\infty}$, we may assume $s_{i}=s_{i}\left(x, y, \vec{z}, x_{1}, y_{1}, \ldots, x_{i-1}, y_{i-1}\right)$ and the same for $t_{i}$. Finally, define $\sigma, \rho, \sigma^{*}, \rho^{*}$ on the term algebra $T(\vec{X})$ with respect to $s_{i}, t_{i}$.

We claim that these $L$ 's, $R$ 's, $s$ 's and $t$ 's satisfy the Mal'cev condition. Let's check it for identity (2.9). Take $\alpha$ with $0<|\alpha|<N$ an even integer. By Lemma 2.1] we have

$$
\varphi=\operatorname{Cg}(\vec{X}, \rho(\vec{X})) .
$$

Since we have $L_{\alpha} \varphi L_{\alpha 1}$ by equation (2.27), Lemma 2.4 gives us terms $p_{i}$ such that for some tuple $\vec{u}, F$ satisfies:

$$
\begin{aligned}
L_{\alpha} & =p_{1}(\vec{X}, \vec{u}), \\
p_{1}(\rho(\vec{X}), \vec{u}) & =p_{2}(\rho(\vec{X}), \vec{u}), \\
p_{2}(\vec{X}, \vec{u}) & =p_{3}(\vec{X}, \vec{u}), \\
& \cdots \\
p_{m}(\rho(\vec{X}), \vec{u}) & =L_{\alpha 1} .
\end{aligned}
$$


Since $\vec{u}=\vec{u}(\vec{X}, \vec{Y})$ can be construed as members of $T\left(Z^{\infty}\right)$, we obtain the following laws for $\mathcal{V}$ :

$$
\begin{aligned}
L_{\alpha}(\vec{X}) & \approx p_{1}(\vec{X}, \vec{u}(\vec{X}, \vec{Y})), \\
p_{1}(\rho(\vec{X}), \vec{u}(\vec{X}, \vec{Y})) & \approx p_{2}(\rho(\vec{X}), \vec{u}(\vec{X}, \vec{Y})), \\
p_{2}(\vec{X}, \vec{u}(\vec{X}, \vec{Y})) & \approx p_{3}(\vec{X}, \vec{u}(\vec{X}, \vec{Y})), \\
& \cdots \\
p_{m}(\rho(\vec{X}), \vec{u}(\vec{X}, \vec{Y})) & \approx L_{\alpha 1}(\vec{X}) .
\end{aligned}
$$

Replacing $\vec{X}$ by $\rho(\vec{X})$ everywhere and noting that $\rho(\rho(\vec{X}))=\rho(\vec{X})$, we have

$$
\begin{aligned}
L_{\alpha}(\rho(\vec{X})) & \approx p_{1}(\rho(\vec{X}), \vec{u}(\rho(\vec{X}), \vec{Y})), \\
p_{1}(\rho(\vec{X}), \vec{u}(\rho(\vec{X}), \vec{Y})) & \approx p_{2}(\rho(\vec{X}), \vec{u}(\rho(\vec{X}), \vec{Y})), \\
p_{2}(\rho(\vec{X}), \vec{u}(\rho(\vec{X}), \vec{Y})) & \approx p_{3}(\rho(\vec{X}), \vec{u}(\rho(\vec{X}), \vec{Y})), \\
& \cdots \\
p_{m}(\rho(\vec{X}), \vec{u}(\rho(\vec{X}), \vec{Y})) & \approx L_{\alpha 1}(\rho(\vec{X})),
\end{aligned}
$$

and by transitivity,

$$
\mathcal{V} \models L_{\alpha}(\rho(\vec{X})) \approx L_{\alpha 1}(\rho(\vec{X})),
$$

which is what we were looking for. The other identities are obtained similarly.

The proof of the previous theorem follows the line of a proof for a Mal'cev condition for BFC. One such condition that closely parallels ours was personally communicated to us by R. Willard.

\section{A Canonical Form of DFC}

We will assume in this section that $\mathcal{V}$ has the Determining Property. Since the Determining Property implies the Left Determining Property, we may define the following formulas in the language of $\mathcal{V}$ :

$$
\Psi_{m}:=\bigwedge_{|\alpha|=m}\left(\left(\bigwedge_{\gamma \neq \varepsilon} L_{\alpha \gamma}(\vec{X})=R_{\alpha \gamma}(\vec{X})\right) \rightarrow L_{\alpha}(\vec{X})=R_{\alpha}(\vec{X})\right),
$$

where every subindex varies over words of length less than or equal to $N$; so an expression of the form " $\bigwedge_{\gamma \neq \varepsilon} L_{\alpha \gamma}=R_{\alpha \gamma}$ " should be read as " $\wedge\left\{L_{\alpha \gamma}=R_{\alpha \gamma}\right.$ : $\gamma \neq \varepsilon$ and $|\alpha \gamma| \leq N\}$ ". Hence $\Psi_{N}=\left(\bigwedge_{|\beta|=N} L_{\beta}(\vec{X})=R_{\beta}(\vec{X})\right)$. (The antecedent "vanishes".)

Lemma 3.1. Let $A \in \mathcal{V}$ and let $\varphi, \varphi^{*} \in \operatorname{Con} A$ such that $\varphi \times \varphi^{*}=\Delta$ and $\overrightarrow{0} \varphi \vec{e} \varphi^{*} \overrightarrow{1}$. Then for all $c, d \in A, A$ satisfies $\Phi_{1}(c, d, \vec{e})$, where

$$
\Phi_{1}(x, y, \vec{z}):=\exists y_{1} \forall x_{1} \ldots \exists y_{n} \forall x_{n} \bigwedge_{m=1}^{k} \Psi_{2 m}
$$

with $n, k$ as in Theorem 2.5 . 
Proof. Take $b_{1}$ such that

$$
s_{1}(c, d, \vec{e}) \stackrel{\varphi}{=} b_{1} \stackrel{\varphi^{*}}{\equiv} t_{1}(c, d, \vec{e}) .
$$

Assuming $b_{i}$ is already chosen and $a_{i}$ is given, define $b_{i+1}$ such that

$$
s_{i+1}\left(c, d, \vec{e}, a_{1}, b_{1}, \ldots, a_{i}, b_{i}\right) \stackrel{\varphi}{=} b_{i+1} \stackrel{\stackrel{\varphi}{*}^{*}}{=} t_{i+1}\left(c, d, \vec{e}, a_{1}, b_{1}, \ldots, a_{i}, b_{i}\right) .
$$

The construction of the $b_{i}$ 's then corresponds to the equations in Corollary 2.3 . Hence, (2.3) implies that $A$ satisfies

$$
\begin{aligned}
& L_{\alpha}\left(c, d, \vec{e}, a_{1}, b_{1}, \ldots, a_{n}, b_{n}\right) \stackrel{\varphi}{=} L_{\alpha}\left(\rho\left(c, d, \vec{e}, a_{1}, b_{1}, \ldots, a_{n}, b_{n}\right)\right), \\
& R_{\alpha}\left(c, d, \vec{e}, a_{1}, b_{1}, \ldots, a_{n}, b_{n}\right) \stackrel{\varphi}{=} R_{\alpha}\left(\rho\left(c, d, \vec{e}, a_{1}, b_{1}, \ldots, a_{n}, b_{n}\right)\right), \\
& L_{\alpha}\left(c, d, \vec{e}, a_{1}, b_{1}, \ldots, a_{n}, b_{n}\right) \stackrel{\varphi^{*}}{=} L_{\alpha}\left(\rho^{*}\left(c, d, \vec{e}, a_{1}, b_{1}, \ldots, a_{n}, b_{n}\right)\right), \\
& R_{\alpha}\left(c, d, \vec{e}, a_{1}, b_{1}, \ldots, a_{n}, b_{n}\right) \stackrel{\varphi^{*}}{=} R_{\alpha}\left(\rho^{*}\left(c, d, \vec{e}, a_{1}, b_{1}, \ldots, a_{n}, b_{n}\right)\right)
\end{aligned}
$$

for all $\alpha$. These together with equations (2.4) imply that for each $\beta$ with $|\beta|=N$,

$$
\begin{aligned}
& L_{\beta}\left(c, d, \vec{e}, a_{1}, b_{1}, \ldots, a_{n}, b_{n}\right) \stackrel{\varphi}{=} R_{\beta}\left(c, d, \vec{e}, a_{1}, b_{1}, \ldots, a_{n}, b_{n}\right), \\
& L_{\beta}\left(c, d, \vec{e}, a_{1}, b_{1}, \ldots, a_{n}, b_{n}\right) \stackrel{\varphi^{*}}{=} R_{\beta}\left(c, d, \vec{e}, a_{1}, b_{1}, \ldots, a_{n}, b_{n}\right) .
\end{aligned}
$$

Since $\varphi \cap \varphi^{*}=\Delta$, this yields $A \models \Psi_{N}\left(c, d, \vec{e}, a_{1}, b_{1}, \ldots, a_{n}, b_{n}\right)$.

Take nonempty $\alpha$ with $|\alpha|<N$ even and suppose

$$
A \models \bigwedge_{\gamma \neq \varepsilon} L_{\alpha \gamma}\left(c, d, \vec{e}, a_{1}, b_{1}, \ldots, a_{n}, b_{n}\right)=R_{\alpha \gamma}\left(c, d, \vec{e}, a_{1}, b_{1}, \ldots, a_{n}, b_{n}\right) .
$$

We can see that $L_{\alpha}\left(c, d, \vec{e}, a_{1}, b_{1}, \ldots, a_{n}, b_{n}\right) \stackrel{\varphi}{=} R_{\alpha}\left(c, d, \vec{e}, a_{1}, b_{1}, \ldots, a_{n}, b_{n}\right)$, by using equations (2.9), (2.10) and (2.11) as follows:

$$
\begin{array}{rlrl}
L_{\alpha}\left(c, d, \vec{e}, a_{1}, b_{1}, \ldots, a_{n}, b_{n}\right) & \stackrel{\varphi}{=} L_{\alpha}\left(\rho\left(c, d, \vec{e}, a_{1}, b_{1}, \ldots, a_{n}, b_{n}\right)\right) & & \text { by (2.3) } \\
& =L_{\alpha 1}\left(\rho\left(c, d, \vec{e}, a_{1}, b_{1}, \ldots, a_{n}, b_{n}\right)\right) & & \text { by (2.9) } \\
& \stackrel{\varphi}{=} L_{\alpha 1}\left(c, d, \vec{e}, a_{1}, b_{1}, \ldots, a_{n}, b_{n}\right) & & \text { by (2.3) } \\
& =R_{\alpha 1}\left(c, d, \vec{e}, a_{1}, b_{1}, \ldots, a_{n}, b_{n}\right) & & \text { by hypothesis } \\
& \stackrel{\varphi}{=} R_{\alpha 1}\left(\rho\left(c, d, \vec{e}, a_{1}, b_{1}, \ldots, a_{n}, b_{n}\right)\right) & & \text { by (2.3) } \\
& =\cdots & & \text { using (2.10) },(3.2) \\
& =\cdots & & \text { and iterating... } \\
& =R_{\alpha k}\left(\rho\left(c, d, \vec{e}, a_{1}, b_{1}, \ldots, a_{n}, b_{n}\right)\right) & \\
& =R_{\alpha}\left(\rho\left(c, d, \vec{e}, a_{1}, b_{1}, \ldots, a_{n}, b_{n}\right)\right) & & \text { using (2.11) } \\
& \stackrel{\varphi}{=} R_{\alpha}\left(c, d, \vec{e}, a_{1}, b_{1}, \ldots, a_{n}, b_{n}\right) & & \text { by (2.3). }
\end{array}
$$

It can be proved in an entirely analogous fashion (by using equations (2.12) that $L_{\alpha}\left(c, d, \vec{e}, a_{1}, b_{1}, \ldots, a_{n}, b_{n}\right) \stackrel{\varphi^{*}}{=} R_{\alpha}\left(c, d, \vec{e}, a_{1}, b_{1}, \ldots, a_{n}, b_{n}\right)$, which yields

$$
A \models L_{\alpha}\left(c, d, \vec{e}, a_{1}, b_{1}, \ldots, a_{n}, b_{n}\right)=R_{\alpha}\left(c, d, \vec{e}, a_{1}, b_{1}, \ldots, a_{n}, b_{n}\right),
$$

and we have proved the lemma. 
Theorem 3.2. Let $\mathcal{V}$ be a variety with the Determining Property, let $A \in \mathcal{V}$ and let $\theta, \theta^{*} \in$ Con $A$ such that $\theta \times \theta^{*}=\Delta$, and $\overrightarrow{0} \theta \vec{e} \theta^{*} \overrightarrow{1}$. Then $c \stackrel{\theta}{\equiv} d$ if and only if $A \models \Phi_{1}(c, d, \vec{e}) \wedge \Phi_{2}(c, d, \vec{e})$, where $\Phi_{1}$ and $\Phi_{2}$ are defined as follows:

$$
\begin{aligned}
\Phi_{1}(x, y, \vec{z}) & :=\exists y_{1} \forall x_{1} \ldots \exists y_{n} \forall x_{n} \bigwedge_{m=1}^{k} \Psi_{2 m}, \\
\Phi_{2}(x, y, \vec{z}) & :=\exists x_{1} \forall y_{1} \ldots \exists x_{n} \forall y_{n} \bigwedge_{m=1}^{k} \Psi_{2 m-1} .
\end{aligned}
$$

Proof. Since the definition of $\Phi_{1}$ is the same as the one given by formula (3.1) in Lemma 3.1 we only have to worry about $\Phi_{2}(c, d, \vec{e})$.

$(\Rightarrow)$ Assume $c \stackrel{\theta}{=} d$. Much in the same way as in the proof of Lemma 3.1, define $a_{1}$ such that

$$
s_{1}(c, d, \vec{e}) \stackrel{\theta}{\equiv} a_{1} \stackrel{\theta^{*}}{\equiv} t_{1}(c, d, \vec{e}) ;
$$

and assuming $a_{i}$ is already chosen and $b_{i}$ is given, let

$$
s_{i+1}\left(c, d, \vec{e}, a_{1}, b_{1}, \ldots, a_{i}, b_{i}\right) \stackrel{\theta}{=} a_{i+1} \stackrel{\theta^{*}}{=} t_{i+1}\left(c, d, \vec{e}, a_{1}, b_{1}, \ldots, a_{i}, b_{i}\right) .
$$

This choice conforms to the pattern of Corollary 2.2. so we obtain

$$
\begin{array}{r}
L_{\alpha}\left(c, d, \vec{e}, a_{1}, b_{1}, \ldots, a_{n}, b_{n}\right) \stackrel{\stackrel{\theta}{\equiv}}{=} L_{\alpha}\left(\sigma\left(c, d, \vec{e}, a_{1}, b_{1}, \ldots, a_{n}, b_{n}\right)\right), \\
R_{\alpha}\left(c, d, \vec{e}, a_{1}, b_{1}, \ldots, a_{n}, b_{n}\right) \stackrel{\theta}{\equiv} R_{\alpha}\left(\sigma\left(c, d, \vec{e}, a_{1}, b_{1}, \ldots, a_{n}, b_{n}\right)\right), \\
L_{\alpha}\left(c, d, \vec{e}, a_{1}, b_{1}, \ldots, a_{n}, b_{n}\right) \stackrel{\theta^{*}}{\equiv} L_{\alpha}\left(\sigma^{*}\left(c, d, \vec{e}, a_{1}, b_{1}, \ldots, a_{n}, b_{n}\right)\right), \\
R_{\alpha}\left(c, d, \vec{e}, a_{1}, b_{1}, \ldots, a_{n}, b_{n}\right) \stackrel{\theta^{*}}{\equiv} R_{\alpha}\left(\sigma^{*}\left(c, d, \vec{e}, a_{1}, b_{1}, \ldots, a_{n}, b_{n}\right)\right)
\end{array}
$$

by equations (2.2).

If we suppose now that

$$
A \models \bigwedge_{\gamma \neq \varepsilon} L_{\alpha \gamma}\left(c, d, \vec{e}, a_{1}, b_{1}, \ldots, a_{n}, b_{n}\right)=R_{\alpha \gamma}\left(c, d, \vec{e}, a_{1}, b_{1}, \ldots, a_{n}, b_{n}\right)
$$

for some $\alpha$ with $|\alpha|<N$ odd, we'll be able to prove $L_{\alpha}\left(c, d, \vec{e}, a_{1}, b_{1}, \ldots, a_{n}, b_{n}\right)=$ $R_{\alpha}\left(c, d, \vec{e}, a_{1}, b_{1}, \ldots, a_{n}, b_{n}\right)$ by showing (in the same way as in Lemma 3.1) that:

- $L_{\alpha}\left(c, d, \vec{e}, a_{1}, b_{1}, \ldots, a_{n}, b_{n}\right) \stackrel{\theta}{\equiv} R_{\alpha}\left(c, d, \vec{e}, a_{1}, b_{1}, \ldots, a_{n}, b_{n}\right)$ (this can be accomplished using (2.13) and (3.3) $)$, and

- $L_{\alpha}\left(c, d, \vec{e}, a_{1}, b_{1}, \ldots, a_{n}, b_{n}\right) \stackrel{\theta^{*}}{\equiv} R_{\alpha}\left(c, d, \vec{e}, a_{1}, b_{1}, \ldots, a_{n}, b_{n}\right)$ (by (2.14) and (3.4) $)$.

$(\Leftarrow)$ Assume $A \models \Phi_{2}(c, d, \vec{e})$. Take $b_{1}$ such that

$$
s_{1}(c, d, \vec{e}) \stackrel{\theta}{\equiv} b_{1} \stackrel{\theta^{*}}{\equiv} t_{1}(c, d, \vec{e}) .
$$

Let $a_{1}$ be given by the outermost existential quantifier of $\Phi_{2}$.

Assuming $b_{i}$ is already chosen and $a_{i}$ is the corresponding witness for $\Phi_{2}$, let

$$
s_{i+1}\left(c, d, \vec{e}, a_{1}, b_{1}, \ldots, a_{i}, b_{i}\right) \stackrel{\theta}{\equiv} b_{i+1} \stackrel{\theta^{*}}{\equiv} t_{i+1}\left(c, d, \vec{e}, a_{1}, b_{1}, \ldots, a_{i}, b_{i}\right) .
$$


This selection conforms to the scheme of Corollary 2.3 (with $\varphi:=\theta$ and $\varphi^{*}:=\theta^{*}$ ) and satisfies the matrix of $\Phi_{1}$, as was seen in the proof of Lemma 3.1. Hence we have, respectively,

$$
\begin{aligned}
& L_{\alpha}\left(c, d, \vec{e}, a_{1}, b_{1}, \ldots, a_{n}, b_{n}\right) \stackrel{\ominus}{\equiv} L_{\alpha}\left(\rho\left(c, d, \vec{e}, a_{1}, b_{1}, \ldots, a_{n}, b_{n}\right)\right), \\
& R_{\alpha}\left(c, d, \vec{e}, a_{1}, b_{1}, \ldots, a_{n}, b_{n}\right) \stackrel{\theta^{*}}{\equiv} R_{\alpha}\left(\rho\left(c, d, \vec{e}, a_{1}, b_{1}, \ldots, a_{n}, b_{n}\right)\right)
\end{aligned}
$$

and

$$
A \models\left(\bigwedge_{m=1}^{N} \Psi_{m}\right)\left(c, d, \vec{e}, a_{1}, b_{1}, \ldots, a_{n}, b_{n}\right) .
$$

From an easy inspection of the form of $\Psi_{m}$, it can be deduced that

$$
A \models \bigwedge_{j=1}^{N} L_{j}\left(c, d, \vec{e}, a_{1}, b_{1}, \ldots, a_{n}, b_{n}\right)=R_{j}\left(c, d, \vec{e}, a_{1}, b_{1}, \ldots, a_{n}, b_{n}\right) .
$$

Therefore,

$$
\begin{aligned}
c & =L_{\varepsilon}\left(c, d, \vec{e}, a_{1}, b_{1}, \ldots, a_{n}, b_{n}\right) & & \text { by (2.5) } \\
& \stackrel{\theta}{=} L_{\varepsilon}\left(\rho\left(c, d, \vec{e}, a_{1}, b_{1}, \ldots, a_{n}, b_{n}\right)\right) & & \text { by (3.5) } \\
& =L_{1}\left(\rho\left(c, d, \vec{e}, a_{1}, b_{1}, \ldots, a_{n}, b_{n}\right)\right) & & \text { by (2.9), with } \alpha=\varepsilon \\
& \stackrel{\theta}{=} L_{1}\left(c, d, \vec{e}, a_{1}, b_{1}, \ldots, a_{n}, b_{n}\right) & & \text { by (3.5) } \\
& =R_{1}\left(c, d, \vec{e}, a_{1}, b_{1}, \ldots, a_{n}, b_{n}\right) & & \text { by (3.6) } \\
& \stackrel{\theta}{\equiv} R_{1}\left(\rho\left(c, d, \vec{e}, a_{1}, b_{1}, \ldots, a_{n}, b_{n}\right)\right) & & \text { by (3.5) } \\
& =L_{2}\left(\rho\left(c, d, \vec{e}, a_{1}, b_{1}, \ldots, a_{n}, b_{n}\right)\right) & & \text { by (2.10) } \\
& \stackrel{\theta}{\equiv} \ldots & & \text { using (2.10) },(3.6) \\
& \stackrel{\theta}{\equiv} \ldots & & \text { and iterating. } \ldots \\
& =R_{N}\left(\rho\left(c, d, \vec{e}, a_{1}, b_{1}, \ldots, a_{n}, b_{n}\right)\right) & & \text { and using (2.11): } \\
& =R_{\varepsilon}\left(\rho\left(c, d, \vec{e}, a_{1}, b_{1}, \ldots, a_{n}, b_{n}\right)\right) & & \\
& \stackrel{\theta}{=} R_{\varepsilon}\left(c, d, \vec{e}, a_{1}, b_{1}, \ldots, a_{n}, b_{n}\right) & & \text { by (3.5) } \\
& =d & & \text { by (2.5). }
\end{aligned}
$$

Hence $c \stackrel{\theta}{\equiv} d$.

\section{Central elements in a variety with DFC}

In the Appendix, we will prove a preservation result (Theorem A.4) that implies that formula $\Phi_{1} \wedge \Phi_{2}$ of Theorem 3.2 is preserved by taking direct factors and direct products (taking $\tau_{\alpha}$ to be " $L_{\alpha}(\vec{X})=R_{\alpha}(\vec{X})$ "). Call $\Phi$ the conjunction $\Phi_{1} \wedge \Phi_{2}$.

Lemma 4.1. Assume $\mathcal{V}$ has the Determining Property. Then there is a set $\Sigma$ of first-order formulas such that for every $A \in \mathcal{V}, \vec{e}, \vec{f} \in A^{l}$ we have that $\vec{e}$ and $\vec{f}$ are complementary central elements if and only if $A=\zeta(\vec{e}, \vec{f})$ for every $\zeta \in \Sigma$. Moreover, each formula in $\Sigma$ is preserved by taking direct factors. 
Proof. The following formulas in the language of $\mathcal{V}$ will assert the properties needed to force $\Phi(\cdot, \cdot, \vec{e})$ and $\Phi(\cdot, \cdot, \vec{f})$ to define the pair of complementary factor congruences associated with $\vec{e}$ and $\vec{f}$.

- $C A N(\vec{e}, \vec{f})=\bigwedge_{i=1}^{l} \Phi\left(0_{i}, e_{i}, \vec{e}\right) \wedge \bigwedge_{i=1}^{l} \Phi\left(1_{i}, f_{i}, \vec{e}\right)$

This formula says that $\vec{e}$ is related to $\overrightarrow{0}$ and $\vec{f}$ to $\overrightarrow{1}$ via $\Phi(\cdot, \cdot, \vec{e})$.

- $\operatorname{PROD}(\vec{e}, \vec{f})=\forall x, y \exists z(\Phi(x, z, \vec{e}) \wedge \Phi(z, y, \vec{f}))$

The relational product of $\Phi(\cdot, \cdot, \vec{e})$ and $\Phi(\cdot, \cdot, \vec{f})$ is the universal congruence.

- $\operatorname{INT}(\vec{e}, \vec{f})=\forall x, y(\Phi(x, y, \vec{e}) \wedge \Phi(x, y, \vec{f}) \rightarrow x=y)$

Their intersection is $\Delta$.

- $R E F(\vec{e}, \vec{f})=\forall x \Phi(x, x, \vec{e})$

$\Phi(\cdot, \cdot, \vec{e})$ is reflexive.

- $S Y M(\vec{e}, \vec{f})=\forall x, y, z(\Phi(x, y, \vec{e}) \wedge \Phi(y, z, \vec{e}) \wedge \Phi(z, x, \vec{f}) \rightarrow z=x)$

- $\operatorname{TRANS}(\vec{e}, \vec{f})=\forall x, y, z, u(\Phi(x, y, \vec{e}) \wedge \Phi(y, z, \vec{e}) \wedge \Phi(x, u, \vec{e}) \wedge \Phi(u, z, \vec{f}) \rightarrow$ $u=z)$

The reader may verify that these two formulas (in conjunction with the previous ones) say that $\Phi(\cdot, \cdot, \vec{e})$ is symmetric and transitive.

- For each $m$-ary function symbol $F$, define:

$$
\begin{aligned}
& P R E S_{F}(\vec{e}, \vec{f})=\forall u_{1}, v_{1}, \ldots, u_{m}, v_{m} \\
& \left(\bigwedge_{j} \Phi\left(u_{j}, v_{j}, \vec{e}\right)\right) \wedge \Phi\left(F\left(u_{1}, \ldots, u_{m}\right), z, \vec{e}\right) \wedge \Phi\left(z, F\left(v_{1}, \ldots, v_{m}\right), \vec{f}\right) \rightarrow \\
& \rightarrow z=F\left(v_{1}, \ldots, v_{m}\right) .
\end{aligned}
$$

These formulas ensure $\Phi(\cdot, \cdot, \vec{e})$ is preserved by the basic operations of $\mathcal{V}$.

Finally, define $C A N^{\prime}, R E F^{\prime}, S Y M^{\prime}, T R A N S^{\prime}$ and $P R E S_{F}^{\prime}$ to be the result of interchanging $\vec{e}$ with $\vec{f}$ in $C A N, R E F, S Y M, T R A N S$ and $P R E S_{F}$, respectively, and let $\Sigma$ be the union of the following two sets:

$$
\left\{C A N, P R O D, I N T, R E F, S Y M, T R A N S, C A N^{\prime}, R E F^{\prime}, S Y M^{\prime}, T R A N S^{\prime}\right\},
$$

$$
\left\{P R E S_{F}, P R E S_{F}^{\prime}: F \text { a function symbol }\right\} \text {. }
$$

Now it is immediate to check that $\vec{e}$ and $\vec{f}$ are complementary central elements if they satisfy all formulas in $\Sigma$. To see the converse, note that if $\vec{e}$ and $\vec{f}$ are complementary central elements, there is an isomorphism $A \rightarrow A_{0} \times A_{1}$ such that $\vec{e}, \vec{f}$ correspond to $[\overrightarrow{0}, \overrightarrow{1}],[\overrightarrow{1}, \overrightarrow{0}]$, respectively, and Theorem 3.2 guarantees that $\Sigma$ will hold.

To see that $\Sigma$ is preserved by direct factors, we first note that each one of $C A N, C A N^{\prime} P R O D, P R O D^{\prime}, R E F$ and $R E F^{\prime}$ is obtained by quantification of a formula preserved by taking direct factors by Theorem A.4. In the second place, the remainder of axioms in $\Sigma$ are of the form $\forall \vec{x}\left(\tau(\vec{e}, \vec{f}, \vec{x}) \rightarrow x_{i}=x_{j}\right)$ with $R E F(\vec{e}, \vec{f}) \wedge R E F^{\prime}(\vec{e}, \vec{f}) \rightarrow \exists \vec{x} \tau(\vec{e}, \vec{f}, \vec{x})$ universally valid, and since $\forall \vec{x}(\tau(\vec{e}, \vec{f}, \vec{x}) \rightarrow$ $\left.x_{i}=x_{j}\right) \wedge \exists \vec{x} \tau(\vec{e}, \vec{f}, \vec{x})$ is preserved by taking direct factors (whenever $\tau(\vec{e}, \vec{f}, \vec{x})$ is preserved by taking direct factors and direct products), we have the result. 
Corollary 4.2. Assume $\mathcal{V}$ has the Determining Property. Then if $\left[\vec{e}_{0}, \vec{e}_{1}\right]$ is a central element of $A_{0} \times A_{1}$, then $\vec{e}_{i}$ is a central element of $A_{i}, i=0,1$.

Proof. Immediate by the previous lemma.

We use $\mathcal{V}_{D I}$ to denote the class of directly indecomposable members of $\mathcal{V}$.

Corollary 4.3. If $\mathcal{V}$ is a variety over a finite language and if it has the Determining Property, then $\mathcal{V}_{D I}$ is axiomatizable by a set of first-order sentences.

Proof. The set $\Sigma=\Sigma(\vec{e}, \vec{f})$ in Lemma 4.1 is finite if the language is finite. Hence

$$
\overrightarrow{0} \neq \overrightarrow{1} \wedge \forall \vec{e}, \vec{f} \bigwedge \Sigma(\vec{e}, \vec{f}) \rightarrow((\vec{e}=\overrightarrow{0} \wedge \vec{f}=\overrightarrow{1}) \vee(\vec{e}=\overrightarrow{1} \wedge \vec{f}=\overrightarrow{0}))
$$

together with axioms for $\mathcal{V}$ defines $\mathcal{V}_{D I}$.

Lemma 4.4. The Determining Property implies BFC.

Proof. By Bigelow and Burris [1, we only need to check that if $A=A_{0} \times A_{1}$, and $\theta$ is a factor congruence on $A$, then

$$
\left\{((a, b),(c, b)): b \in A_{1} \text { and } \exists a^{\prime}, c^{\prime}\left(a, a^{\prime}\right) \theta\left(c, c^{\prime}\right)\right\} \subseteq \theta .
$$

Let $\vec{e}=\left[\vec{e}_{0}, \vec{e}_{1}\right]$ be the central element associated to $\theta$, so " $x \theta y$ " is defined by $\Phi(x, y, \vec{e})$. We have

$$
\left(a, a^{\prime}\right) \theta\left(c, c^{\prime}\right) \text { iff } A_{0} \times A_{1} \models \Phi\left(\left(a, a^{\prime}\right),\left(c, c^{\prime}\right),\left[\vec{e}_{0}, \vec{e}_{1}\right]\right) .
$$

By Theorem A.4, this implies

$$
A_{0} \models \Phi\left(a, c, \vec{e}_{0}\right) .
$$

Now Corollary 4.2 ensures $\vec{e}_{1}$ is central in $A_{1}$, and hence $A_{1} \models \Phi\left(b, b, \vec{e}_{1}\right)$. Since $\Phi$ is preserved by direct products, we obtain

$$
A_{0} \times A_{1}=\Phi\left((a, b),(c, b),\left[\vec{e}_{0}, \vec{e}_{1}\right]\right),
$$

and then $(a, b) \theta(c, b)$.

\section{THE MAIN THEOREM}

Proof of Theorem 1.1. (5) $\Rightarrow(2)$ Suppose we have a pair of complementary factor congruences $\varphi$ and $\varphi^{*}$ such that $\overrightarrow{0} \varphi \vec{e} \varphi^{*} \overrightarrow{1}$. Suppose now that also $\theta \times \theta^{*}=\Delta$ and $\overrightarrow{0} \theta \vec{e} \theta^{*} \overrightarrow{1}$. Then $\overrightarrow{0} \stackrel{\varphi \circ \theta^{*}}{\equiv} \overrightarrow{1}$ and hence $\varphi \vee \theta^{*}=\nabla$. So we have

$$
\left(\varphi \vee \theta^{*}\right) \cap \theta=\theta \text {. }
$$

By BFC, we obtain $\varphi \cap \theta=\theta$ and then $\varphi \subseteq \theta$. By symmetry, we obtain $\varphi=\theta$ and $\varphi^{*}=\theta^{*}$.

$(2) \Rightarrow(3)$ Theorem 3.2 ,

$(3) \Rightarrow(4)$ Obvious.

$(4) \Rightarrow(1)$ Immediate.

$(2) \Rightarrow(5)$ Lemma 4.4

$(1) \Rightarrow(5)$ Define $\tilde{0}_{i}$ and $\tilde{1}_{i}$ with $i=1, \ldots, 2 l$ in the following way:

$$
\begin{aligned}
\left(\tilde{0}_{1}, \ldots, \tilde{0}_{2 l}\right) & :=\left(0_{1}, \ldots 0_{l}, 1_{1}, \ldots 1_{l}\right), \\
\left(\tilde{1}_{1}, \ldots, \tilde{1}_{2 l}\right): & =\left(1_{1}, \ldots 1_{l}, 0_{1}, \ldots, 0_{l}\right) .
\end{aligned}
$$

It can be easily checked (using the Weak Determining Property) that with these $\tilde{0}$ 's and $\tilde{1}$ 's we have the Determining Property. Since $(2) \Rightarrow(5)$, we have our result. 


\section{EXAMPLES}

6.1. $\overrightarrow{0} \& \overrightarrow{1}$ does not imply BFC. The variety $\mathcal{V}_{L}$ with language $\{+, *, 0,1\}$ given by the following set of equations $\Sigma$ :

$$
\begin{aligned}
x+0 & =x, \\
x+1 & =x * 1, \\
x * 0 & =0
\end{aligned}
$$

has $\overrightarrow{0} \& \overrightarrow{1}$. Next we will define various algebras in $\mathcal{V}_{L}$. In the first place take $L_{\omega}:=\langle\omega,+, *, 0,1\rangle$, where

$$
\begin{array}{ll}
0+1:=0, & 0 * 1:=0, \\
1+1:=1, & 1 * 1:=1, \\
x+0:=x, & x * 0:=0
\end{array}
$$

for all $x \in \omega$ and

$$
z+y:=2, \quad z * y:=2
$$

for all $z, y \in \omega$ not previously considered. For each $n \geq 2, L_{n}$ will denote the subalgebra of $L_{\omega}$ with universe $n=\{0,1, \ldots, n-1\}$. Now define $D_{n}$ to be the subalgebra of $L_{2} \times L_{\omega}$ with universe $(2 \times n) \cup\{(1, n)\}$.

Define the following subsets of $2 \times \omega$ :

$$
\begin{aligned}
& P_{0}:=\{(0, j) \mid 3 \leq j\}, \\
& P_{1}:=\{(1, j) \mid 3 \leq j\} .
\end{aligned}
$$

Then $2 \times \omega=(2 \times 3) \cup P_{0} \cup P_{1}$. Note that for all $z \in(2 \times \omega) \backslash\{(0,0),(1,0)\}$ and for all $x, y \in P_{1}$ we have:

$$
\begin{aligned}
x+z & =y+z, \\
z+x & =z+y, \\
x * z & =y * z, \\
z * x & =z * y .
\end{aligned}
$$

Lemma 6.1. Every injective partial function $f: D_{n} \rightarrow\left(L_{2} \times L_{n}\right)$ which fixes $(2 \times 3) \cup P_{0}$ is a partial isomorphism between $D_{n}$ and $L_{2} \times L_{n}$.

Proof. It's straightforward to see (using equations (6.1)) that if $B \subseteq P_{1}$ and $\sigma$ is any permutation of $P_{1}$, then $(2 \times 3) \cup P_{0} \cup B$ and $(2 \times 3) \cup P_{0} \cup \sigma(B)$ are subalgebras of $L_{2} \times L_{\omega}$ and

$$
\bar{\sigma}(x):= \begin{cases}x & x \in(2 \times 3) \cup P_{0}, \\ \sigma(x) & x \in B\end{cases}
$$

is an isomorphism between them.

Since $f$ is a restriction of such an isomorphism $\bar{\sigma}$, it is a partial isomorphism.

Recall that $\left(\mathcal{V}_{L}\right)_{D I}$ is the class of directly indecomposable members of $\mathcal{V}_{L}$.

Lemma 6.2. Let $\mathcal{V}$ be a variety. If $\mathcal{V}_{D I}$ is axiomatizable by a set of first-order sentences, then it is finitely axiomatizable relative to $\mathcal{V}$.

Proof. First note that an ultraproduct of directly decomposable algebras is again decomposable. Let $\Sigma$ be a set of first-order sentences axiomatizing $\mathcal{V}_{D I}$. By way of contradiction, suppose that $\mathcal{V}_{D I}$ is not finitely axiomatizable relative to $\mathcal{V}$. Hence, 
for each finite $\Sigma_{0} \subseteq \Sigma$ there exists $A_{\Sigma_{0}} \in \mathcal{V} \backslash \mathcal{V}_{D I}$ satisfying $\Sigma_{0}$. Now it is easy to construct an ultraproduct $U$ of these decomposable algebras in such a way that $U$ satisfies $\Sigma$, an absurdity.

Theorem 6.3. $\left(\mathcal{V}_{L}\right)_{D I}$ is not axiomatizable by a set of first-order sentences.

Proof. We will first prove that player " $\exists$ " has a winning strategy for the back-andforth (or "Ehrenfeucht") game of length $n-3$ played on $D_{n}$ and $L_{2} \times L_{n}$. The strategy is as follows:

- If $\forall$ chooses an element in $(2 \times 3) \cup P_{0}$ (in either algebra), $\exists$ will choose the same element in the other algebra.

- If $\forall$ chooses an element in $P_{1}, \exists$ will choose an element in the $P_{1}$-part of the other algebra, which has not been chosen up to this point.

There are $n-3$ elements in $P_{1} \cap\left(L_{2} \times L_{n}\right)$, so these instructions work up to $n-3$ moves. Let's call $g$ the partial function defined by this game. By Lemma 6.1, $g$ is a partial isomorphism and we have proved our first claim.

Now suppose $\varphi$ is a sentence such that $\left(\mathcal{V}_{L}\right)_{D I} \models \varphi$. By the above strategy we have that for every sufficiently large $n, D_{n} \models \varphi$ if and only if $L_{2} \times L_{n} \models \varphi$. By taking $n$ such that $2 n+1=$ cardinal of $D_{n}$ is a prime number, we obtain $D_{n} \in\left(\mathcal{V}_{L}\right)_{D I}$. We conclude that there are decomposable algebras satisfying $\varphi$, and hence $\left(\mathcal{V}_{L}\right)_{D I}$ cannot be defined by a single first-order sentence. Using Lemma 6.2 we have our result.

Corollary 6.4. $\mathcal{V}_{L}$ does not have DFC.

Proof. Since DFC is equivalent to the Determining Property, we can use Corollary 4.3 and Theorem 6.3 .

An indication that $\left(\mathcal{V}_{L}\right)_{D I}$ might not be definable was discovered by using the "Universal Algebra Calculator" program, designed by Ralph Freese and Emil Kiss [6].

6.2. Semilattice expansions. Throughout this section, we will suppose that $\mathcal{V}$ is a variety with $\overrightarrow{0} \& \overrightarrow{1}$ for which there exists a binary term $\vee$ such that for every $A \in \mathcal{V}, \vee^{A}$ is a semilattice operation on $A$. We will keep the assumption that the language of $\mathcal{V}$ has at least one constant.

First, we observe that by Lemma 2.4 together with the observation that $(x, y) \in$ $\nabla^{F}=\operatorname{Cg}^{F}(\overrightarrow{0}, \overrightarrow{1})$ (where $F \in \mathcal{V}$ is the free algebra freely generated by $\{x, y\}$ ), we obtain $(2+l)$-ary terms $u_{i}(x, y, \vec{z}), i=1, \ldots, k$, such that the following identities hold in $\mathcal{V}$ :

$$
\begin{aligned}
x & \approx u_{1}(x, y, \overrightarrow{0}), \\
u_{i}(x, y, \overrightarrow{1}) & \approx u_{i+1}(x, y, \overrightarrow{1}) \text { with } i \text { odd, } \\
u_{i}(x, y, \overrightarrow{0}) & \approx u_{i+1}(x, y, \overrightarrow{0}) \text { with } i \text { even, } \\
u_{k}(x, y, \overrightarrow{1}) & \approx y
\end{aligned}
$$

Proposition 6.5. The formula

$$
\Phi(x, y, \vec{z}):=\forall u\left(\bigwedge_{i=1}^{k}\left(u_{i}(x, y, \overrightarrow{0}) \vee u=u_{i}(x, y, \vec{z}) \vee u\right) \quad \longrightarrow \quad(x \vee u=y \vee u)\right)
$$

satisfies (3) of Theorem 1.1 for $\mathcal{V}$. 
Proof. Let $A, B \in \mathcal{V}$, and $a \in A, b, d \in B$. First we prove that

$$
A \times B \models \Phi((a, b),(a, d),[\overrightarrow{0}, \overrightarrow{1}]) .
$$

Suppose that for some $(u, v)$ we have

$$
A \times B \models \bigwedge_{i=1}^{k} u_{i}((a, b),(a, d),[\overrightarrow{0}, \overrightarrow{0}]) \vee(u, v)=u_{i}((a, b),(a, d),[\overrightarrow{0}, \overrightarrow{1}]) \vee(u, v) .
$$

Then

$$
B \models \bigwedge_{i=1}^{k} u_{i}(b, d, \overrightarrow{0}) \vee v=u_{i}(b, d, \overrightarrow{1}) \vee v .
$$

But the above equations in combination with (6.2) produce

$$
b \vee v=d \vee v
$$

and hence

$$
(a, b) \vee(u, v)=(a, d) \vee(u, v)
$$

Now suppose

$$
A \times B=\Phi((a, b),(c, d),[\overrightarrow{0}, \overrightarrow{1}]) .
$$

The reader can check that considering $u=\left(a, \bigvee_{i=1}^{k} u_{i}(b, d, \overrightarrow{0}) \vee u_{i}(b, d, \overrightarrow{1})\right)$, it can be proved that $a \vee c=a$, and similarly with $u=\left(c, \bigvee_{i=1}^{k} u_{i}(b, d, \overrightarrow{0}) \vee u_{i}(b, d, \overrightarrow{1})\right)$ and $a \vee c=c$; hence $a=c$.

The following example will show that the complexity of formula $\Phi$ in the above proposition cannot be improved for the general case.

Proposition 6.6. Let $\mathcal{V}^{\vee}$ be the variety with language $\{+, *, 0,1, \vee\}$ defined by the axioms of $\mathcal{V}_{L}$ plus identities saying that $\vee$ is a semilattice operation for which $0 \vee 1=0$. Then there exists neither a positive nor an existential formula satisfying (4) of Theorem 1.1 for $\mathcal{V}^{\vee}$.

Proof. Define join-semilattice operations on $L_{2}, L_{4}$ and $L_{5}$ such that they are totally ordered with the order given by $0>1>2>3>4$. Suppose that $\Phi$ satisfies (4) of Theorem 1.1, and consider $L_{5} \times L_{2}$.

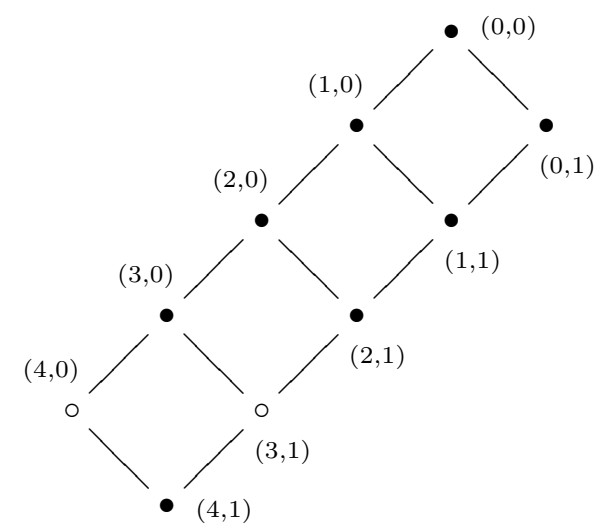

FiguRE 1. The semilattice structure of $L_{5} \times L_{2}$. 
The shaded dots in Figure 1 form a subalgebra of $L_{5} \times L_{2}$; call it $L$. The reader may check that $F: L_{4} \times L_{2} \rightarrow L$, where

$$
F(x):= \begin{cases}x & x \neq(3,1), \\ (4,1) & x=(3,1)\end{cases}
$$

is an isomorphism. Since $\Phi$ witnesses (4) of Theorem 1.1 for $\mathcal{V}^{\vee}$, we have

$$
L_{4} \times L_{2}=\Phi((3,0),(3,1),(0,1),(1,0)) .
$$

Applying $F$ everywhere,

$$
L \models \Phi((3,0),(4,1),(0,1),(1,0)) .
$$

If we had $\Phi$ an existential formula, we would obtain

$$
L_{5} \times L_{2}=\Phi((3,0),(4,1),(0,1),(1,0)),
$$

since $L$ is a subalgebra of $L_{5} \times L_{2}$. We would then conclude $3=4$, an absurdity.

The fact that there is no positive formula $\Phi$ is an immediate consequence of the next two claims. We say that $\mathcal{V}$ has compact factor congruences if every factor congruence of every algebra in $\mathcal{V}$ is compact.

Claim 1. $\mathcal{V}^{\vee}$ does not have compact factor congruences.

Proof. If $\mathcal{V}^{\vee}$ had compact factor congruences, there would exist $\mathbf{0}_{1}(w), \ldots, \mathbf{0}_{N}(w)$, $\mathbf{1}_{1}(w), \ldots, \mathbf{1}_{N}(w)$ such that for every algebra $A=A_{1} \times A_{2} \in \mathcal{V},\left(\lambda_{1}, \lambda_{2}\right) \in A$,

$$
\begin{aligned}
& \operatorname{ker} \pi_{1}=\operatorname{Cg}^{A}\left(\left[\overrightarrow{\mathbf{0}}\left(\lambda_{1}\right), \overrightarrow{\mathbf{0}}\left(\lambda_{2}\right)\right],\left[\overrightarrow{\mathbf{0}}\left(\lambda_{1}\right), \overrightarrow{\mathbf{1}}\left(\lambda_{2}\right)\right]\right), \\
& \operatorname{ker} \pi_{2}=\operatorname{Cg}^{A}\left(\left[\overrightarrow{\mathbf{1}}\left(\lambda_{1}\right), \overrightarrow{\mathbf{1}}\left(\lambda_{2}\right)\right],\left[\overrightarrow{\mathbf{0}}\left(\lambda_{1}\right), \overrightarrow{\mathbf{1}}\left(\lambda_{2}\right)\right]\right),
\end{aligned}
$$

by Lemma 4 in [13. Since the language contains constants, we can replace these new $\mathbf{0}$ 's and $\mathbf{1}$ 's by closed terms, and hence

$$
\begin{aligned}
& \operatorname{ker} \pi_{1}=\operatorname{Cg}^{A}([\overrightarrow{\mathbf{0}}, \overrightarrow{\mathbf{0}}],[\overrightarrow{\mathbf{0}}, \overrightarrow{\mathbf{1}}]), \\
& \operatorname{ker} \pi_{2}=\operatorname{Cg}^{A}([\overrightarrow{\mathbf{1}}, \overrightarrow{\mathbf{1}}],[\overrightarrow{\mathbf{0}}, \overrightarrow{\mathbf{1}}]) .
\end{aligned}
$$

Now, checking the axioms of $\mathcal{V}^{\vee}$, we conclude that for every closed term $t$ in the language of $\mathcal{V}^{\vee}, t$ is equivalent to 0 or $t$ is equivalent to 1 over $\mathcal{V}^{\vee}$; hence we should have

$$
\operatorname{ker} \pi_{1}=\mathrm{Cg}^{A}((0,0),(0,1)) \vee \mathrm{Cg}^{A}((1,0),(1,1)) .
$$

But the reader may check that if we take $A=L_{5} \times L_{2}$, the equivalence relation depicted in Figure 2 is a congruence that contains the right hand side of the last equality and is clearly different from $\operatorname{ker} \pi_{1}$.

Claim 2. Let $\mathcal{V}$ be a variety with $\overrightarrow{0} \& \overrightarrow{1}$. Suppose that $\Phi$ is a positive formula that satisfies (4) of Theorem 1.1. Then $\mathcal{V}$ has compact factor congruences.

Proof. Let $A \in \mathcal{V}$. We will prove that if $\varphi \times \varphi^{*}=\Delta, \overrightarrow{0} \varphi \vec{e} \varphi^{*} \overrightarrow{1}$ and $\overrightarrow{1} \varphi \vec{f} \varphi^{*} \overrightarrow{0}$, then $\varphi=\operatorname{Cg}(\overrightarrow{0}, \vec{e}) \vee \operatorname{Cg}(\overrightarrow{1}, \vec{f})$, and hence is compact. Call $\theta:=C g(\overrightarrow{0}, \vec{e}) \vee \operatorname{Cg}(\overrightarrow{1}, \vec{f})$. Trivially, $\theta \subseteq \varphi$. Assume $x \varphi y$; by (4) of Theorem 1.1 we obtain $A=\Phi(x, y, \vec{e}, \vec{f})$. Since $\Phi$ is positive, it is preserved by homomorphic images and then

$$
A / \theta \models \Phi(x / \theta, y / \theta, \vec{e} / \theta, \vec{f} / \theta) .
$$

Equivalently,

$$
A / \theta \models \Phi(x / \theta, y / \theta, \overrightarrow{0} / \theta, \overrightarrow{1} / \theta),
$$




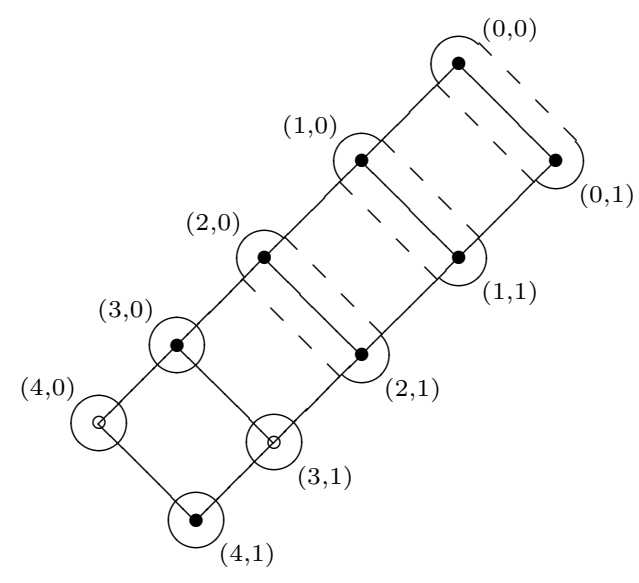

Figure 2. One congruence in $L_{5} \times L_{2}$.

and we obtain $x / \theta=y / \theta$. This implies $(x, y) \in \theta$, hence $\varphi \subseteq \theta$, and we have the result.

\section{Final CONSIDERATIONS}

We wish to mention that Theorem 1.1 is in some sense a consequence of Beth's definability theorem of first-order logic. This is because the first evidence that the Determining Property implies Definable Factor Congruences was obtained by a simple application of this theorem. Once we are sure about the concrete existence of a certain first-order formula 1 we know that all efforts dedicated to finding it are not sterile. Due to the expressive power of terms in algebra, it is common that first-order definitions obtained in this area are more manageable than in the general case of model theory, and this fact makes the task of searching for them a little easier.

Another tool that was important for our research for this paper, but was not included in the final presentation, is the Pierce sheaf. Working on this construction, we realized that there was some sense in developing a structural study of central elements when factor congruences are not compact.

Finally, we are indebted to Ross Willard for the terms $s_{i}(\ldots)$ and $t_{i}(\ldots)$, which appear in his (unpublished) version of a Mal'cev condition for Boolean Factor Congruences.

\section{Appendix A. A preservation Result}

For the rest of the section, $N$ will be an even natural number.

Lemma A.1. For every word $\alpha$ in the alphabet $\{1, \ldots, N\}$ of length no greater than $N$, let $\tau_{\alpha}=\tau_{\alpha}\left(x, y, \vec{z}, x_{1}, y_{1}, \ldots, x_{n}, y_{n}\right)$ be a formula preserved by direct products and by taking direct factors. Define:

$$
E_{m}:=\bigwedge_{\substack{m \leq|\alpha| \leq N \\|\alpha| \text { even }}}\left(\bigwedge_{\gamma \neq \varepsilon} \tau_{\alpha \gamma} \rightarrow \tau_{\alpha}\right), \quad O_{m}:=\bigwedge_{\substack{m \leq|\alpha| \leq N \\|\alpha| \text { odd }}}\left(\bigwedge_{\gamma \neq \varepsilon} \tau_{\alpha \gamma} \rightarrow \tau_{\alpha}\right)
$$

\footnotetext{
${ }^{1}$ As is well known, the formulas given by Beth's theorem can be effectively found.
} 
Then,

(1) For $2 \leq m \leq N$, $m$ even, if

$$
\left(\exists y_{1} \forall x_{1} \ldots \exists y_{n} \forall x_{n} E_{m}\right) \wedge\left(\exists x_{1} \forall y_{1} \ldots \exists x_{n} \forall y_{n} O_{m+1}\right)
$$

is preserved by direct factors, so is

$$
\left(\exists y_{1} \forall x_{1} \ldots \exists y_{n} \forall x_{n} E_{m}\right) \wedge\left(\exists x_{1} \forall y_{1} \ldots \exists x_{n} \forall y_{n} O_{m-1}\right) .
$$

(2) For $4 \leq m \leq N$, $m$ even, if

$$
\left(\exists y_{1} \forall x_{1} \ldots \exists y_{n} \forall x_{n} E_{m}\right) \wedge\left(\exists x_{1} \forall y_{1} \ldots \exists x_{n} \forall y_{n} O_{m-1}\right)
$$

is preserved by direct factors, so is

$$
\left(\exists y_{1} \forall x_{1} \ldots \exists y_{n} \forall x_{n} E_{m-2}\right) \wedge\left(\exists x_{1} \forall y_{1} \ldots \exists x_{n} \forall y_{n} O_{m-1}\right) .
$$

Note that every subindex varies over words of length less than or equal to $N$, so an expression of the form " $\bigwedge_{\gamma \neq \varepsilon} \tau_{\alpha \gamma}$ " should be read as " $\wedge\left\{\tau_{\alpha \gamma}: \gamma \neq \varepsilon\right.$ and $|\alpha \gamma| \leq$ $N\}$ ". Therefore, if $m \geq N, O_{m}=$ true (empty conjunction) and $E_{N}=\bigwedge_{|\beta|=N} \tau_{\beta}$. Also, recall that the $i$-th component of an element $a$ in a direct product $\Pi_{i} A_{i}$ is called $a^{i}$.

We will now state and prove two lemmas that will be helpful in order to prove Lemma A.1. In the following we will assume that the tuple $\vec{z}$ has length equal to 1 , since proofs are exactly the same and this simplification makes them easier to read.

Lemma A.2. Let $m$ be an even integer, $A_{0}, A_{1} \in \mathcal{V}$ and $c, d, e, a_{1}, \ldots, a_{2 n} \in$ $A_{0} \times A_{1}$ such that $2 \leq m \leq N, A_{0} \times A_{1} \models E_{m}\left(c, d, e, a_{1}, \ldots, a_{2 n}\right)$ and $A_{1} \models$ $O_{m+1}\left(c^{1}, d^{1}, e^{1}, a_{1}^{1}, \ldots, a_{2 n}^{1}\right)$. Then $A_{0} \models E_{m}\left(c^{0}, d^{0}, e^{0}, a_{1}^{0}, \ldots, a_{2 n}^{0}\right)$ and if $\alpha$ has length $m$, then

$A_{0}=\left(\bigwedge_{\gamma \neq \varepsilon} \tau_{\alpha \gamma}\right)\left(c^{0}, d^{0}, e^{0}, a_{1}^{0}, \ldots, a_{2 n}^{0}\right) \Rightarrow A_{0} \times A_{1} \models\left(\bigwedge_{\mu} \tau_{\alpha \mu}\right)\left(c, d, e, a_{1}, \ldots, a_{2 n}\right)$.

Proof. By induction on $m$. If $m=N$, the first part is immediate since $E_{N}$ is a conjunction of formulas preserved by taking direct factors and hence preserved by taking direct factors. The second part is contained in the hypothesis.

To make the proof more readable, we will omit the string of parameters. Take an even $m$ such that $2 \leq m<N$ and suppose the lemma is proved for $m+2$. Assume

$$
\begin{aligned}
& A_{0} \times A_{1} \models E_{m}, \quad \text { noting that } \quad E_{m}=E_{m+2} \wedge \bigwedge_{|\alpha|=m}\left(\bigwedge_{\gamma \neq \varepsilon} \tau_{\alpha \gamma} \rightarrow \tau_{\alpha}\right) ; \\
& A_{1} \models O_{m+1}, \quad \text { noting that } \quad O_{m+1}=O_{m+3} \wedge \bigwedge_{|\alpha|=m+1}\left(\bigwedge_{\gamma \neq \varepsilon} \tau_{\alpha \gamma} \rightarrow \tau_{\alpha}\right) .
\end{aligned}
$$

By the first part of the inductive hypothesis we thus have $A_{0} \models E_{m+2}$. We have to see that $A_{0} \models \bigwedge_{|\alpha|=m}\left(\bigwedge_{\gamma \neq \varepsilon} \tau_{\alpha \gamma} \rightarrow \tau_{\alpha}\right)$. Suppose now that for some $\alpha$ of length $m$,

$$
A_{0} \models \bigwedge_{\gamma \neq \varepsilon} \tau_{\alpha \gamma}
$$


In particular, for each $i, j \leq N$ we have

$$
A_{0} \models \bigwedge_{\gamma \neq \varepsilon} \tau_{\alpha i j \gamma}
$$

We will prove that $A_{0} \models \tau_{\alpha}$, and the second part of the lemma will be proved along the way.

By the second part of the inductive hypothesis (i.e., (A.1) ) we obtain, for all $j$,

$$
A_{0} \times A_{1} \models \bigwedge_{\mu} \tau_{\alpha i j \mu} \quad \text { or, in other symbols, } \quad A_{0} \times A_{1} \models \bigwedge_{\gamma \neq \varepsilon} \tau_{\alpha i \gamma} .
$$

Since this last formula is preserved by taking direct factors, we have

$$
A_{1} \models \bigwedge_{\gamma \neq \varepsilon} \tau_{\alpha i \gamma}
$$

Using (A.3) (note $|\alpha i|=m+1$ ), we have $A_{1} \models \tau_{\alpha i}$ for all $i$. This, together with (A.5) yields $A_{1} \models \bigwedge_{\gamma \neq \varepsilon} \tau_{\alpha \gamma}$. Now we apply (A.4), thus obtaining

$$
A_{0} \times A_{1} \models \bigwedge_{\gamma \neq \varepsilon} \tau_{\alpha \gamma}
$$

Applying (A.2),

$$
A_{0} \times A_{1} \models \tau_{\alpha} .
$$

The last two formulas jointly say

$$
A_{0} \times A_{1} \models\left(\bigwedge_{\mu} \tau_{\alpha \mu}\right) .
$$

We have proved $($ A.4 $) \Rightarrow$ A.6), which is the second conclusion. Since $\tau_{\alpha}$ is preserved by taking direct factors, we obtain $A_{0} \models \tau_{\alpha}$, which is the first conclusion.

Lemma A.3. Let $m$ be an even integer, $A_{0}, A_{1} \in \mathcal{V}$ and $c, d, e, a_{1}, \ldots, a_{2 n} \in$ $A_{0} \times A_{1}$ such that $2 \leq m \leq N, A_{0} \times A_{1} \models O_{m-1}\left(c, d, e, a_{1}, \ldots, a_{2 n}\right)$ and $A_{1} \models$ $E_{m}\left(c^{1}, c^{1}, e^{1}, a_{1}^{1}, \ldots, a_{2 n}^{1}\right)$. Then $A_{0}=O_{m-1}\left(c^{0}, d^{0}, e^{0}, a_{1}^{0}, \ldots, a_{2 n}^{0}\right)$ and if $\alpha$ has length $m-1$, then

$A_{0} \models\left(\bigwedge_{\gamma \neq \varepsilon} \tau_{\alpha \gamma}\right)\left(c^{0}, d^{0}, e^{0}, a_{1}^{0}, \ldots, a_{2 n}^{0}\right) \Rightarrow A_{0} \times A_{1} \models\left(\bigwedge_{\mu} \tau_{\alpha \mu}\right)\left(c, d, e, a_{1}, \ldots, a_{2 n}\right)$.

Proof. By induction on $m$. If $m=N$, the hypotheses are:

$$
\begin{aligned}
A_{0} \times A_{1} \models O_{N-1} & =\bigwedge_{|\alpha|=N-1}\left(\bigwedge_{\gamma \neq \varepsilon} \tau_{\alpha \gamma} \rightarrow \tau_{\alpha}\right), \\
A_{1} \models E_{N} & =\bigwedge_{|\beta|=N} \tau_{\beta} .
\end{aligned}
$$

Assume that for some $\alpha$ of length $N-1$,

$$
A_{0} \models \bigwedge_{\gamma \neq \varepsilon} \tau_{\alpha \gamma}=\bigwedge_{i} \tau_{\alpha i}
$$

By using (A.8) and preservation by direct products, we have

$$
A_{0} \times A_{1} \models \bigwedge \tau_{\alpha i} .
$$

By (A.7) we have $A_{0} \times A_{1}=\tau_{\alpha}$, thus obtaining

$$
A_{0} \times A_{1} \models \bigwedge_{\mu} \tau_{\alpha \mu}
$$


We have proved the second part of the lemma. Passing to $A_{0}$ we obtain the first part.

Now take an even $m$ such that $2 \leq m<N$ and suppose the lemma is proved for $m+2$. Assume

$$
\begin{aligned}
A_{0} \times A_{1} \models O_{m-1} & =O_{m+1} \wedge \bigwedge_{|\alpha|=m-1}\left(\bigwedge_{\gamma \neq \varepsilon} \tau_{\alpha \gamma} \rightarrow \tau_{\alpha}\right), \\
A_{1} \models E_{m} & =E_{m+2} \wedge \bigwedge_{|\alpha|=m}\left(\bigwedge_{\gamma \neq \varepsilon} \tau_{\alpha \gamma} \rightarrow \tau_{\alpha}\right) .
\end{aligned}
$$

By the inductive hypothesis we thus have $A_{0} \models O_{m+1}$. The rest of the argument closely parallels the proof of Lemma A.2.

Proof of Lemma A.1. To see (11), suppose

$$
A_{0} \times A_{1} \models\left(\left(\exists y_{1} \forall x_{1} \ldots \exists y_{n} \forall x_{n} E_{m}\right) \wedge\left(\exists x_{1} \forall y_{1} \ldots \exists x_{n} \forall y_{n} O_{m-1}\right)\right)(c, d, e) .
$$

Thus we have (Skolem) functions $G_{1}, \ldots, G_{n}$ such that $G_{i}$ is $(i-1)$-ary and

$$
A_{0} \times A_{1} \models \forall \vec{y} O_{m-1}\left(c, d, e, G_{1}, y_{1}, \ldots, G_{n}\left(y_{1}, \ldots, y_{n-1}\right), y_{n}\right) .
$$

Since $O_{m-1}$ implies $O_{m+1}$, we have

$$
A_{0} \times A_{1} \models\left(\left(\exists y_{1} \forall x_{1} \ldots \exists y_{n} \forall x_{n} E_{m}\right) \wedge\left(\exists x_{1} \forall y_{1} \ldots \exists x_{n} \forall y_{n} O_{m+1}\right)\right)(c, d, e) .
$$

Then, since this formula is preserved by taking direct factors, by hypothesis,

$$
\begin{aligned}
& A_{0} \models\left(\exists y_{1} \forall x_{1} \ldots \exists y_{n} \forall x_{n} E_{m}\right)\left(c^{0}, d^{0}, e^{0}\right), \\
& A_{1} \models\left(\exists y_{1} \forall x_{1} \ldots \exists y_{n} \forall x_{n} E_{m}\right)\left(c^{1}, d^{1}, e^{1}\right) .
\end{aligned}
$$

Thus we have functions $F_{1}, \ldots, F_{n}$ such that

$$
A_{1} \models \forall \vec{x} E_{m}\left(c^{1}, d^{1}, e^{1}, x_{1}, F_{1}, \ldots, x_{n}, F_{n}\left(x_{1}, \ldots, x_{n-1}\right)\right) .
$$

Now, for $j=1, \ldots, n$, define $j$-ary functions $p_{j}$ from $A_{0}$ to $A_{0} \times A_{1}, p_{j}=$ $p_{j}\left(a_{1}, \ldots, a_{j}\right)$ :

$$
\begin{aligned}
& p_{1}:=\left(a_{1}, F_{1}\right), \\
& p_{2}:=\left(a_{2}, F_{2}\left(G_{1}^{1}\right)\right), \\
& p_{j}:=\left(a_{j}, F_{j}\left(G_{1}^{1}, G_{2}\left(p_{1}\right)^{1}, \ldots, G_{j-1}\left(p_{1}, \ldots, p_{j-2}\right)^{1}\right)\right) .
\end{aligned}
$$

The reader may check that this selection ensures, for each $\vec{a} \in A_{0}^{n}$ :

$$
\begin{aligned}
A_{0} \times A_{1} & \models O_{m-1}\left(c, d, e, G_{1}, p_{1}, \ldots, G_{n}\left(p_{1}, \ldots, p_{n-1}\right), p_{n}\right), \\
A_{1} & =E_{m}\left(c^{1}, d^{1}, e^{1}, G_{1}^{1}, p_{1}^{1}, \ldots, G_{n}\left(p_{1}, \ldots, p_{n-1}\right)^{1}, p_{n}^{1}\right) .
\end{aligned}
$$

We may apply Lemma A.3 and obtain

$$
A_{0} \models O_{m-1}\left(c^{0}, d^{0}, e^{0}, G_{1}^{0}, p_{1}^{0}, \ldots, G_{n}\left(p_{1}, \ldots, p_{n-1}\right)^{0}, p_{n}^{0}\right) .
$$

Equivalently,

$$
A_{0} \models O_{m-1}\left(c^{0}, d^{0}, e^{0}, G_{1}^{0}, a_{1}, G_{2}\left(p_{1}\right)^{0}, a_{2}, \ldots, G_{n}\left(p_{1}, \ldots, p_{n-1}\right)^{0}, a_{n}\right) .
$$


Now, defining $H_{j}: A_{0}^{j-1} \rightarrow A_{0}$ as follows:

$$
\begin{aligned}
H_{1} & :=G_{1}^{0}, \\
H_{2}\left(y_{1}\right) & :=G_{2}\left(p_{1}\left(y_{1}\right)\right)^{0}, \\
H_{j}\left(y_{1}, \ldots, y_{j-1}\right) & :=G_{j}\left(p_{1}\left(y_{1}\right), \ldots, p_{j-1}\left(y_{1}, \ldots, y_{j-1}\right)\right)^{0},
\end{aligned}
$$

we see at once that

$$
A_{0} \models \forall \vec{y} O_{m-1}\left(c^{0}, d^{0}, e^{0}, H_{1}, y_{1}, \ldots, H_{n}\left(y_{1}, \ldots, y_{n-1}\right), y_{n}\right),
$$

and then

$$
A_{0} \models\left(\exists x_{1} \forall y_{1} \ldots \exists x_{n} \forall y_{n} O_{m-1}\right)\left(c^{0}, d^{0}, e^{0}\right) .
$$

This, together with (A.9), proves this case.

Part (2) is entirely analogous to the former, and it's proved by using Lemma A.2,

The hypotheses of the next theorem are the same as in Lemma A.1 and we repeat them for ease of reference.

Theorem A.4. For every word $\alpha$ in the alphabet $\{1, \ldots, N\}$ of length no greater than $N$, let $\tau_{\alpha}=\tau_{\alpha}\left(x, y, \vec{z}, x_{1}, y_{1}, \ldots, x_{n}, y_{n}\right)$ be a formula preserved by taking direct products and direct factors. Define:

$$
E_{m}:=\bigwedge_{\substack{m \leq|\alpha| \leq N \\|\alpha| \text { even }}}\left(\bigwedge_{\gamma \neq \varepsilon} \tau_{\alpha \gamma} \rightarrow \tau_{\alpha}\right), \quad O_{m}:=\bigwedge_{\substack{m \leq|\alpha| \leq N \\|\alpha| \text { odd }}}\left(\bigwedge_{\gamma \neq \varepsilon} \tau_{\alpha \gamma} \rightarrow \tau_{\alpha}\right) .
$$

Then the formula

$$
\left(\exists y_{1} \forall x_{1} \ldots \exists y_{n} \forall x_{n} E_{2}\right) \wedge\left(\exists x_{1} \forall y_{1} \ldots \exists x_{n} \forall y_{n} O_{1}\right)
$$

is preserved by taking direct factors and direct products.

Proof. First observe that

$$
\left(\exists y_{1} \forall x_{1} \ldots \exists y_{n} \forall x_{n} E_{N}\right) \wedge\left(\exists x_{1} \forall y_{1} \ldots \exists x_{n} \forall y_{n} O_{N+1}\right)=\exists y_{1} \forall x_{1} \ldots \exists y_{n} \forall x_{n} \bigwedge_{|\beta|=N} \tau_{\beta}
$$

is preserved by direct factors. This is immediate since the conjunction and quantification of formulas preserved by taking direct factors is again preserved by taking direct factors. Successive application of Lemma A.1 yields that A.11) is preserved by taking direct factors.

The proof that (A.11) is preserved by direct products is a straightforward calculation.

\section{REFERENCES}

1. D. Bigelow and S. Burris, Boolean algebras of factor congruences, Acta Sci. Math. (Szeged) 54 (1990), 11-20. MR1073415 (91j:08012)

2. S. BuRRIS, Boolean products of indecomposables, Algebra Universalis 48 no. 2 (2002), 497-499. MR.1967096

3. C. C. Chang, B. Jónsson and A. TARski, Refinement properties for relational structures. Fund. Math. 54 (1964), 249-281. MR0172811(30:3029)

4. S. Comer, Representations by algebras of sections of Boolean Spaces, Pacific J. Math. 38 (1971), 29-38. MR0304277 (46:3412)

5. B. A. DaveY, Sheaf spaces and sheaves of universal algebras, Math Z., 134 (1973), 275-290. MR 0330006 (48:8345) 
6. R. Freese And E. KISS, An algebra calculator program. Website: http://www.math.hawaii. edu/ ralph/software/uaprog/

7. B. Jónsson And A. TARSKI, Direct Decompositions of Finite Algebraic Systems. University of Notre Dame, South Bend, IN (1947). MR0020543 (8:560b)

8. R. McKenzie, G. McNulty and W. Taylor, Algebras, Lattices, Varieties, Volume 1, The Wadsworth \& Brooks/Cole Math. Series, Monterey, California (1987).

9. P. Krauss and D. Clark, Global subdirect products, Mem. Amer. Math. Soc. 210 (1979). MR512475 (80b:08001)

10. R. S. PiERCE, Modules over commutative regular rings, Mem. Amer. Math. Soc. 70 (1967). MR.0217056 (36:151)

11. A. TARSKI, Cardinal Algebras. Oxford Univ. Press, New York (1949). MR0029954 (10:686f)

12. D. VAGGione, Central elements in varieties with the Fraser-Horn property, Advances in Mathematics 148 (1999), 193-202. MR1736957 (2001b:08005)

13. D. Vaggione And P. SÁnchez Terraf, Compact factor congruences imply Boolean factor congruences, Algebra Universalis 51 (2004) 207-213. MR2099798(2005f:08001)

14. R. Willard, Varieties Having Boolean Factor Congruences. J. Algebra, 132 (1990), 130-153. MR.1060837 (91i:08010)

Ciem - Facultad de Matemática, Astronomía y Física (Fa.M.A.F.), Universidad Nacional de Córdoba, Ciudad Universitaria, Córdoba 5000, Argentina

E-mail address: sterraf@famaf .unc.edu.ar

CiEM - Facultad de Matemática, Astronomía y Física (Fa.M.A.F.), Universidad Nacional de Córdoba, Ciudad Universitaria, Córdoba 5000, Argentina

E-mail address: vaggione@mate.uncor.edu 